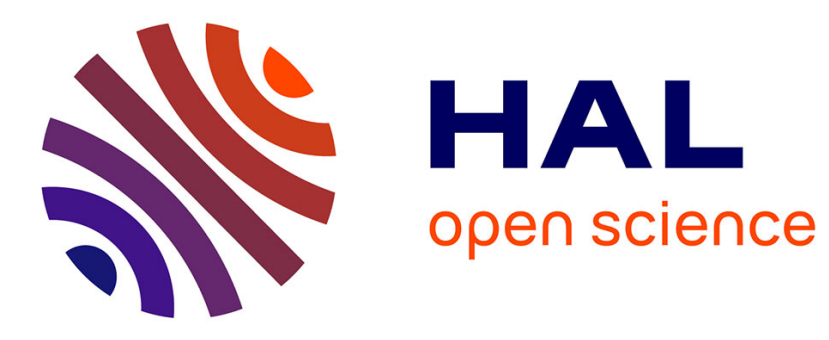

\title{
Linear instability, nonlinear instability, and ligament dynamics in three-dimensional laminar two-layer liquid/liquid flows
}

Lennon O Naraigh, Prashant Valluri, David M. Scott, Iain Bethune, Peter D.M. Spelt

\section{To cite this version:}

Lennon O Naraigh, Prashant Valluri, David M. Scott, Iain Bethune, Peter D.M. Spelt. Linear instability, nonlinear instability, and ligament dynamics in three-dimensional laminar two-layer liquid/liquid flows. Journal of Fluid Mechanics, 2014, 750, pp.464-506. 10.1017/jfm.2014.274 . hal-01044603

\section{HAL Id: hal-01044603 https://hal.science/hal-01044603}

Submitted on 23 Jul 2014

HAL is a multi-disciplinary open access archive for the deposit and dissemination of scientific research documents, whether they are published or not. The documents may come from teaching and research institutions in France or abroad, or from public or private research centers.
L'archive ouverte pluridisciplinaire HAL, est destinée au dépôt et à la diffusion de documents scientifiques de niveau recherche, publiés ou non, émanant des établissements d'enseignement et de recherche français ou étrangers, des laboratoires publics ou privés. 


\title{
Linear instability, nonlinear instability, and ligament dynamics in three-dimensional laminar two-layer liquid/liquid flows
}

\author{
By LENNON Ó NÁRAIGH ${ }^{1}$, PRASHANT VALLURI ${ }^{2}$, \\ DAVID M. SCOT T ${ }^{3}$, IAIN BETHUNE \\ PETER D. M. SPEL T
}

\author{
${ }^{1}$ School of Mathematical Sciences, University College Dublin, Belfield, Dublin 4, Ireland $\dagger$ \\ ${ }^{2}$ Institute of Materials and Processes, Sanderson Building, School of Engineering, University of \\ Edinburgh, King's Buildings, Edinburgh, EH9 3JL, UK \\ ${ }^{3}$ Edinburgh Parallel Computing Centre, The University of Edinburgh, United Kingdom \\ ${ }^{4}$ Département Mécanique, Université de Lyon 1 and Laboratoire de Mécanique des Fluides \& \\ d'Acoustique (LMFA), CNRS, Ecole Centrale Lyon, Ecully, France
}

(Received May 12, 2014)

We consider the linear and nonlinear stability of two-phase density-matched but viscositycontrasted fluids subject to laminar Poiseuille flow in a channel, paying particular attention to the formation of three-dimensional waves. A combination of Orr-SommerfeldSquire analysis (both modal and non-modal) with direct numerical simulation of the three-dimensional two-phase Navier-Stokes equations is used. For the parameter regimes under consideration, under linear theory, the most unstable waves are two-dimensional. Nevertheless, we demonstrate several mechanisms whereby three-dimensional waves enter the system, and dominate at late time. There exists a direct route, whereby threedimensional waves are amplified by the standard linear mechanism; for certain parameter classes, such waves grow at a rate less than but comparable to that of most-dangerous two-dimensional mode. Additionally, there is a weakly nonlinear route, whereby a purely spanwise wave grows according to transient linear theory and subsequently couples to a streamwise mode in weakly nonlinear fashion. Consideration is also given to the ultimate state of these waves: persistent three-dimensional nonlinear waves are stretched and distorted by the base flow, thereby producing regimes of ligaments, 'sheets', or 'interfacial turbulence'. Depending on the parameter regime, these regimes are observed either in isolation, or acting together.

\section{Introduction}

Two-layer channel flows are a useful model for several industrial systems, including oil/gas transport and the cleaning of surfaces by flow. A large body of literature is devoted to the linear theory of infinitesimally small perturbations in these flows, mostly on (periodic) two-dimensional perturbations in the streamwise and wall-normal directions of a uni-directional base state. The subject of this paper is the route by which three-dimensional small-amplitude waves eventually leads to wave overturning, ligament formation and droplet entrainment allowing for flows that are not periodic in the main flow direction. We concentrate herein on density-matched laminar systems, representative of liquid/liquid flows, with the view that the additional effects of a density contrast 
is best studied subsequently; a viscosity contrast is anyway usually present in gas/liquid systems, and is known often to produce the dominant mechanism for linear instability, as recalled below. Our objective is accomplished using a high-resolution direct numerical simulation of two-phase density-matched but viscosity-contrasted flows in a long channel.

One possible route to droplet formation is a purely linear one: a single linear mode dominates and holds up to a very late stage before ligament formation. Prior work on linear stabililty analysis of two-layer channel flows has been mostly on two-dimensional systems, within the framework of Orr-Sommerfeld theory. Results obtained with twodimensional nonlinear direct numerical simulations in Valluri et al. $(2007,2010)$ follow linear theory up to a point close to the turnover of waves. Linear stability analysis has revealed that the dominant mechanism that leads to linear temporal growth is the socalled Yih mechanism (Yiantsios \& Higgins 1988), due to the viscosity contrast across the interface (Yih 1967). Viscosity stratification leads to net work being done by the perturbation velocity and stress at the interface. By using an energy budget, Boomkamp \& Miesen (1996) verified that this mechanism plays an important role in many papers on interfacial instability. Other mechanisms for instability are also conveniently summarized by Boomkamp \& Miesen (1996). Of particular importance for laminar two-layer flow here also is a Tollmien-Schlichting or shear-type mechanism (possibly in both fluids). Competition between shear modes and the Yih mode has been observed for particular choices of flow parameters (Yecko et al. 2002). The extension of the modal analysis to threedimensional disturbances, although not straightforward in view of the fact that Squire's theorem does not necessarily apply (Yiantsios \& Higgins 1988), shows that the dominant mode is two-dimensional in a wide range of multiphase flow situations, albeit that for some parameter values large-amplitude three-dimensional waves might result (Sahu \& Matar 2011).

The present work is focused on laminar pressure-driven channel flow where both viscosity-contrast modes and shear modes are present (as in the classification of Boomkamp \& Miesen (1996)). For completeness we mention briefly the mechanism of Miles (1957) that pertains in turbulent flows. There, the transfer of energy from the mean flow into the wave perturbations is governed by the sign of the second derivative of the base-state flow at the critical layer - the height where the wave speed and the base-state velocity match. For viscosity-driven instabilities (exemplified by the study of Yih (1967)), the critical layer plays no role in the generation of instability, despite re-emerging as an important factor in the case of miscible systems: for a continuously-stratified viscosity profile, Craik (1969) showed for a Couette-type flow, the viscosity stratification induces a curvature in the velocity profile which, if negative at the critical layer, promotes instability. We emphasize however that the present work is focused exclusively on immiscible systems with a piecewise constant viscosity profile. The reader is referred to the review by Govindarajan \& Sahu (2014) for a more in-depth discussion of miscible flows, as well as three-layer viscosity stratification.

In practice, disturbances are expected initially to be localized, and modal temporal stability analysis may be of restricted value, as the manner whereby such initially localized pulses are amplified should really be considered in linearly unstable cases: either amplification in at least one moving frame of reference and damping in the laboratory frame (convective instability), or growing disturbances in the entire domain in the laboratory frame (absolute instability). To determine absolute instability from the OrrSommerfeld theory for the two-dimensional model, the saddle-point method was used initially in Valluri et al. (2010); Ó Náraigh et al. (2013) but difficulties arose due to the presence of singularities in the complex wavenumber plane and due to spatio-temporal mode competition. It was therefore necessary to confirm independently the boundaries 
between convectively and absolutely unstable cases in parameter space. For this purpose, an alternative approach was developed in by Ó Náraigh et al. (2013) wherein the OrrSommerfeld eigenvalue problem was converted into a Cauchy problem such that highlyefficient DNS of the linearized equations of motion can be performed, and the evolution of an initially-localized pulse tracked (the so-called ray-analysis approach, based on earlier work on single-phase flows (Delbende \& Chomaz 1998; Delbende et al. 1998)). Using this approach, the evolution of a pulse in an arbitrary frame of reference travelling at velocity $v$ with respect to the laboratory frame can be tracked, and the pulse growth rate obtained as a function of $v$, from which the convectively or absolutely unstable nature of instability can then be concluded. The existing evidence (Sahu \& Matar 2011) is that allowing for three-dimensional disturbances does not significantly move convective/absolute boundaries here.

The extent to which such linear theory governs three-dimensional wave growth has not yet been established. Furthermore, the growth rates from a modal linear analysis are merely asymptotic (i.e. valid in the notional limit as $t \rightarrow \infty$ but before the onset of nonlinear effects). Effects not captured by a modal analysis can be present at early times and lead to substantial transient growth because the Orr-Sommerfeld equation is non-normal and the eigenfunctions of a given mode are not orthogonal. In this way, the growth of an arbitrarily-chosen initial condition containing a mixture of Orr-Sommerfeld eigenmodes can produce transient growth rates that are orders of magnitude in excess of the asymptotic growth rates computed from the standard eigenvalue analyses. Transient growth has been reported for the two-dimensional two-layer channel-flow problem (van Noorden et al. 1998) and for transverse modes in a two-layer mixing layer (Yecko \& Zaleski 2005).

Various nonlinear mechanisms may eventually become dominant. For instance, the linearly-most-dangerous streamwise mode may interact weakly nonlinearly with spanwise modes, and the evolution of the spanwise modes is 'slaved' via a centre-manifold-type approximation to the streamwise spanwise mode. For generic references to this theory, see the work by Schmid \& Henningson (2001). A specific version of this theory also exists, but concerns only streamwise (two-dimensional) modes in the context of a longwave model that only approximates the underlying equations of motion (Barthelet et al. 1995). An extension of this approach has been carried out by King \& McCready (2000), and accounts for a range of of weakly nonlinear interactions between a wide variety of modes that are strongly excited in the linear theory. The work by King \& McCready (2000) is based on the full linearized Navier-Stokes equations, but is still focused only on streamwise (two-dimensional) modes.

The linearly-most-dangerous streamwise mode may stabilize at large amplitude and thereafter, a finite-amplitude unidirectional travelling wave superimposed on the base state is treated as a 'new' base state. This new base state may be unstable to threedimensional perturbations. Typically, secondary growth rates are computed via Floquet analysis (Schmid \& Henningson 2001). For gas/liquid jet flows, such a route has been identified by Marmottant \& Villermaux (2004), involving a secondary instability of RayleighTaylor type. Although the present study is on density-matched systems, ligament growth is observed in the results below, such that there must be mechanisms other than the Rayleigh-Taylor instability at work here.

Once a wave has overturned to form a ligament, various scenarios may lead to droplets. A tentative study of three-dimensional two-phase mixing-layer flow by Scardovelli \& Zaleski (1999) found that initial conditions corresponding to two-dimensional linear theory cause the interface to develop into 'sheets', which break to form cylinders that subsequently break up due to capillary instability. On the other hand, for initial conditions that 
are sufficiently far from the description given by linear theory, spanwise waves were found to develop on the sheet edges that further develop into cylinders - this time pointing in the streamwise direction - which then break up. Sheets and ligaments are observed in jet breakup as well as in two-layer flows, with sheets often inflated resulting in a bag-type shape in gas/liquid systems (e.g. the work by Marmottant \& Villermaux (2004)).

Various governing mechanisms of the evolution of three-dimensional sheets and ligaments have been identified. In the work by Marmottant \& Villermaux (2004), a simple force balance was proposed as a model of ligament dynamics in gas-assisted jet breakup: mainly form drag exerted by the gas flow was argued to lead to an increase in momentum of the ligament. A different mechanism has been identified in breakup of droplets pinned on an adhering surface by shear flow in density-matched systems: there, the work done by the tangential stress exerted by the exterior fluid is converted into surface energy (Ding et al. 2010).

This paper is organized as follows. After an overview of methodology and validation tests in Section 2, and an outline of the pertinent general behaviour of modal and nonmodal linear growth in Section 3, we use direct numerical simulations of channel flows in a periodic domain in Section 4 to establish to what extent linear theory is followed, or whether a non-linear mechanism becomes significant before the turnover of waves. In Section 5 we investigate 'open' domains (with an inlet and an outlet). Ligament dynamics are investigated in Section 6, wherein we also report a transition to a highly-agitated flow regime. Concluding remarks are presented in Section 7.

\section{Problem statement and computational methodologies}

In this section we present the problem statement and the computational methodologies used in this study, which are linear theory and direct numerical simulation (DNS) of the fully nonlinear governing equations, using the twophase levelset solver (TPLS).

\subsection{Problem statement}

Throughout this paper, we study the two-phase Navier-Stokes equations, in a rectangular Cartesian frame $(x, y, z)$ in a channel geometry $\left[0, L_{x}\right] \times\left[0, L_{y}\right] \times\left[0, L_{z}\right]$. We fix $L_{z}=1$ in the nondimensional framework described below. We are interested in density-matched pressure-driven channel flow, for which an equilibrium configuration is unidirectional two-phase Poiseuille flow, wherein the phases are confined in two layers parallel to the direction of flow. Either the pressure gradient or the flow rate is fixed. We therefore assign labels $j=B, T$ to the fluid layers, and consider the case in which the bottom layer contains the more viscous fluid. The dimensionless physical parameters in the problem are the Reynolds number $R e=\rho_{T} V L_{z} / \mu_{T}$, the viscosity ratio $m=\mu_{B} / \mu_{T}$ (bottom/top), the surface-tension parameter (inverse capillary number) $\mathcal{S}=\gamma /\left(\mu_{T} V\right)$. Here, $\gamma$ denotes the (dimensional) surface tension. Across interfaces separating the two phases, the following standard jump conditions are satisfied:

$$
\llbracket \widehat{\boldsymbol{n}} \cdot\left[-p \mathbb{I}+\mu_{j}\left(\nabla \boldsymbol{u}_{j}+\nabla \boldsymbol{u}_{j}^{T}\right)\right] \rrbracket \cdot \widehat{\boldsymbol{t}}^{(r)}=0, \quad \llbracket \widehat{\boldsymbol{n}} \cdot\left[-p \mathbb{I}+\mu_{j}\left(\nabla \boldsymbol{u}_{j}+\nabla \boldsymbol{u}_{j}^{T}\right)\right] \cdot \widehat{\boldsymbol{n}} \rrbracket=\mathcal{S} \kappa,
$$

where $\widehat{\boldsymbol{n}}$ is a normal vector to the interface (pointing from $j=B$ to $j=T$ ), and $\widehat{\boldsymbol{t}}^{(1)}$ and $\widehat{\boldsymbol{t}}^{(2)}$ are the tangent vectors. The brackets $\llbracket \cdot \rrbracket$ denote the jump condition across the interface $((j=T)-(j=B))$, and $\kappa$ denotes the interfacial (mean) curvature. Note that for large-amplitude non-equilibrium situations (characterized by wave overturning and droplet entrainment), the notion of 'top' and 'bottom' fluids becomes ambiguous. 


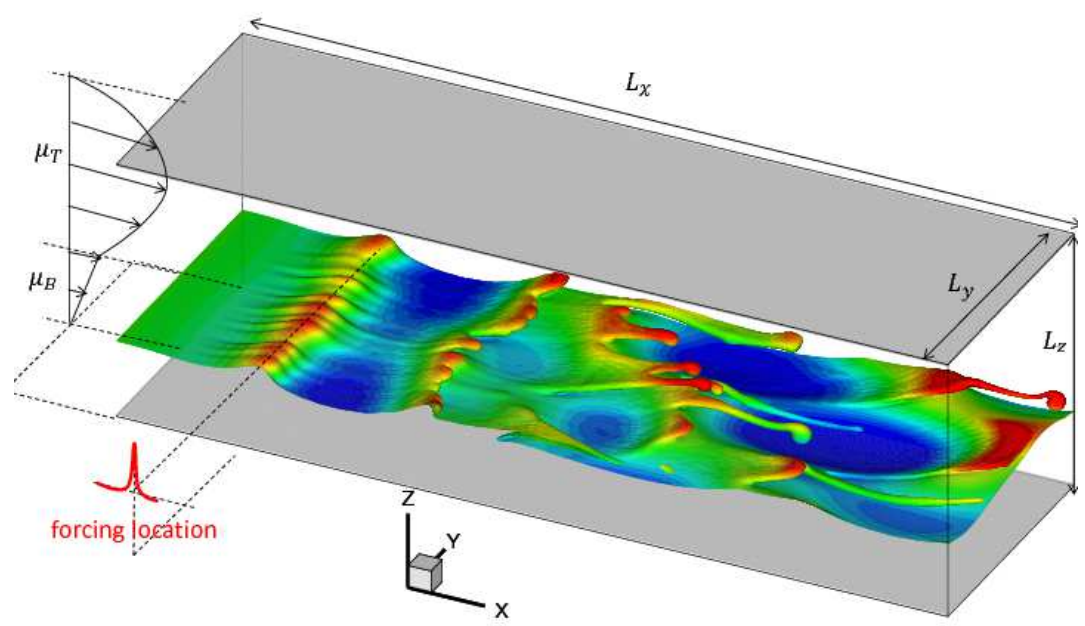

Figure 1. Definition sketch showing the open-flow case. Periodic conditions are used in the $y$-direction. The interface shown is a result of a simulation that is discussed in Section 6 (the interface has been coloured by the local height of the interface location).

However, the ambiguity is removed by identifying $j=T$ with the less viscous fluid and $j=B$ with its more viscous counterpart.

Boundary conditions are required to close the system of equations. Bounding walls with the implied no-slip boundary conditions are introduced at $z=0$ and $z=L_{z}$. Inlet/outlet boundary conditions ('open flow') are imposed in the streamwise $(x$-) direction, and periodic boundary conditions in the spanwise $(y-)$ direction: at the inlet, the velocity field is prescribed as $\boldsymbol{u}(x=0)=\left(U_{0}(z), 0,0\right)$, and at the outlet, $\partial_{x} \boldsymbol{u}=0$. Here $U_{0}(z)$ is a prescribed inlet condition, the mean value of which sets the velocity scale $V$. Throughout this paper, the inlet condition is taken to be Poiseuille flow-profile obtained by computing the steady flat-interface solution of the Navier-Stokes equations for a particular flatinterface height $h_{0}$. At times, we also make use of periodic boundary conditions in the $x$-direction. Finally, the system is perturbed either through initial conditions or through a forcing localized in space; details of these are presented together with the linear and nonlinear simulation techniques in the subsequent subsections. A definition sketch for the open-flow case is shown in Figure 1; the periodic case is similar.

\subsection{Linear theory}

Linear theory is used in subsequent sections to study the early-time development of threedimensional disturbances. It is shown that some of the characteristics of the linear regime are retained at later times by the subsequent nonlinear regime, thereby underscoring the importance of understanding fully the initial linear phase of the wave development. In linear theory, small-amplitude disturbances grow or decay exponentially, with growth rates and phase speeds determined from a modal Orr-Sommerfeld-Squire (OSS) eigenvalue analysis. The idea behind this approach is to linearize the equations of motion around a steady base state corresponding to uni-directional flow, to reduce the linearized equations down to an equation pair involving the wall-normal velocity and vorticity, and to 
Laplace-Fourier transform the resulting linear differential-algebraic equation. The transformed problem is recalled here in generic terms as follows:

$$
\lambda\left(\begin{array}{cc}
\mathcal{M}_{O S} & 0 \\
\mathcal{M}_{C} & \mathcal{M}_{S}
\end{array}\right)\left(\begin{array}{c}
\widetilde{w}_{\alpha \beta}(z, \lambda) \\
\widetilde{\omega}_{z, \alpha \beta}(z, \lambda)
\end{array}\right)=\left(\begin{array}{cc}
\mathcal{L}_{O S} & 0 \\
\mathcal{L}_{C} & \mathcal{L}_{S}
\end{array}\right)\left(\begin{array}{c}
\widetilde{w}_{\alpha \beta}(z, \lambda) \\
\widetilde{\omega}_{z, \alpha \beta}(z, \lambda)
\end{array}\right),
$$

where $\mathcal{L}_{O S}$ depends on wavenumbers and the wall-normal derivative, $\mathcal{L}_{O S}=\mathcal{L}_{O S}\left[\mathrm{i} \alpha, \mathrm{i} \beta, \partial_{z}\right]$, and similarly for the other operators. Here, following standard notation, $\alpha$ denotes a wavenumber in the streamwise direction and $\beta$ denotes a wavenumber in the spanwise direction. Also, the base state implicit in Equation (2.2) is the analytically-computed Poiseuille profile got from the Navier-Stokes equations by balancing the pressure drop with the viscous stress. For wall-bounded flows, solution of Equation (2.2) for the eigenvalue $\lambda$ gives a discrete family of eigenvalues for each Fourier mode, $\left\{\lambda_{n}(\alpha, \beta)\right\}_{n=0}^{\infty}$. The growth rate is then determined by the eigenvalue with the largest real part. In fact, this approach is rather standard and is not discussed further here (but see Sahu \& Matar (2011) and Appendix A; the analytic base state is also provided in the same appendix).

The eigenvalue probem (2.2) can be viewed as the Laplace-transform of a differentialalgebraic equation, the asymptotic solution of which picks out the most-dangerous eigenmode of Equation (2.2). However, at finite times, a combination of modes can combine to produce transient growth rates in excess of the asymptotic most-dangerous exponential growth rate. This is possible because the eigenfunctions of Equation (2.2) are non-orthogonal, which is due in turn to the non-normality of the operators in the same equation (Trefethen et al. 1993; Schmid \& Henningson 2001). The resulting transient growth is captured by the maximum amplification factor

$$
G_{\alpha \beta}(t)=\sup \left\|\left(w_{\alpha \beta}(z, t), \omega_{z, \alpha \beta}(z, t)\right)\right\|_{E},
$$

where $\|\cdot\|_{E}$ denotes the energy norm (Schmid \& Henningson 2001; van Noorden et al. $1998)$, and where the supremum is taken over all possibile states $\left(w_{\alpha \beta}(t), \omega_{\alpha \beta}(t)\right)$ whose energy norm at $t=0$ is unity. Computation of the maximum amplification factor is standard, and is carred out in this work in a manner similar to that used by Yecko \& Zaleski (2005).

\subsection{Nonlinear DNS}

Beyond linear theory, DNS of the Navier-Stokes equations is used in the following, through the stage of overturning waves, ligament formation and up to the point of droplet entrainment. A levelset method is utilized with a continuous surface tension model (Sussman \& Fatemi 1998). In this levelset formalism, the basic Navier-Stokes equations for density-matched fluids are modelled as

$$
\begin{gathered}
\left(\frac{\partial \boldsymbol{u}}{\partial t}+\boldsymbol{u} \cdot \nabla \boldsymbol{u}\right)=-\nabla p+\frac{1}{R e} \nabla \cdot\left[\mu\left(\nabla \boldsymbol{u}+\nabla \boldsymbol{u}^{T}\right)\right]+\delta_{\epsilon}(\phi) \mathcal{S} \hat{\boldsymbol{n}} \nabla \cdot \hat{\boldsymbol{n}}, \\
\nabla \cdot \boldsymbol{u}=0, \\
\hat{\boldsymbol{n}}=\frac{\nabla \phi}{|\nabla \phi|}, \quad \frac{\partial \phi}{\partial t}+\boldsymbol{u} \cdot \nabla \phi=0 .
\end{gathered}
$$

Here, $\phi(\boldsymbol{x}, t)$ is the levelset function indicating in which phase the point $\boldsymbol{x}$ lies $(\phi<0$ in the bottom layer, $\phi>0$ in the top layer). The (possibly multivalued) interface $\eta(\boldsymbol{x}, t)$ is therefore the zero level set, $\phi(\boldsymbol{x}, t)=0 \Longrightarrow \boldsymbol{x}=(x, y, \eta(x, y, t))$. Moreover, the levelset function determines the unit vector normal to the interface $(\hat{\boldsymbol{n}})$, as well as the viscosity, via the relation $\mu=m\left(1-H_{\epsilon}(\phi)\right)+H_{\epsilon}(\phi)$. The function $H_{\epsilon}(\phi)$ is a regularized Heaviside function, which is smooth across a width $\epsilon=1.5 \Delta x$. Finally, $\delta_{\epsilon}(s)=d H_{\epsilon}(s) / d s$ is a 
regularized delta function supported on an interval $[-\epsilon, \epsilon]$. The delta function $\delta_{\epsilon}(s)$ is implemented either via an analytical expression for $d H_{\epsilon}(s) / d s$, or through finite-differencing of $H_{\epsilon}(\cdot)$. For the problem under consideration, both approaches yield identical results. The details of the numerical method are discussed below under several headings.

Grid structure, momentum treatment: The velocities and pressure are discretized on an isotropic MAC grid, with velocities defined at cell faces and pressures defined at cell centres, and grid spacing $\Delta z$; the timestep is denoted by $\Delta t$. The convective derivative is treated using a third-order Adams-Bashforth scheme (Boyd 2001). The momentum fluxes are treated in a flux-conservative fashion, and the derivatives in the momentum term in the $i^{\text {th }}$ direction in Equation (2.4a) are written as follows:

$$
\underbrace{\partial_{x}\left(\mu \partial_{x} u_{i}\right)+\partial_{y}\left(\mu \partial_{y} u_{i}\right)+\partial_{z}\left(\mu \partial_{z} u_{i}\right)}_{=\mathcal{D}_{i}}+\underbrace{\partial_{x}\left(\mu \partial_{x_{i}} u\right)+\partial_{y}\left(\mu \partial_{x_{i}} v\right)+\partial_{z}\left(\mu \partial_{x_{i}} w\right)}_{=\mathcal{C}_{i}}
$$

such that each derivative in the sum can be approximated numerically as a difference taken between two cell faces, thereby accurately taking account of the momentum flux between cells. The expression (2.5) is discretized in time using a Crank-Nicolson treatment for the second-order manifestly diffusive terms $\mathcal{D}_{i}$ and a third-order Adams-Bashforth treatment for the leftover terms $\mathcal{C}_{i}$ :

$$
\frac{1}{2}\left(\mathcal{D}_{i}^{n+1}+\mathcal{D}_{i}^{n}\right)+\frac{23}{12} \mathcal{C}_{i}^{n}-\frac{4}{3} \mathcal{C}_{i}^{n-1}+\frac{5}{12} \mathcal{C}_{i}^{n-2},
$$

where $n$ here denotes the $n^{\text {th }}$ timestep in the simulation. Several other methods for implementing the finite-differencing of the momentum term were attempted, but the method described here proved to be the best at capturing the stress profile across the interface.

Pressure treatment, operator inversions, levelset advection: The pressure and associated incompressibility constraint are treated using the projection method. Both the implicit momentum step and the pressure correction step call for Helmholtz's equation and Poisson's equation, respectively. For the Helmholtz step, successive over-relaxation is used. For the Poisson step, we experimented with two methods: successive overrelaxation, or GMRES with a block-Jacobi preconditioner, which was implemented using the PETSc library. The two methods yielded identical results, albeit that the code's performance was substantially improved under GMRES (Scott et al. 2013a). Finally, the levelset function $\phi$ is advected using a third-order WENO scheme (Ding et al. 2007), and the resulting updated levelset function is reinitialized using a Hamilton-Jacobi equation and the algorithm of Russo \& Smereka (2000). The maximum curvature is limited to $1 / \Delta z$.

Practical implementation: The numerical method is coded in Fortran 90 for implementation on a distributed/shared-memory architecture, using a combination of OpenMP and MPI (Gropp et al. 1994). The code is run on a supercomputer in which the basic processor is an AMD Opteron 2.3GHz Interlagos processor. For a typical simulation 1024 such processors were used for 12 hours. The source code ('TPLS') of the GMRES version is available under an open-source license (Scott et al. (2013b)).

This approach resolves large changes in interfacial topology with only a small amount of mass loss. Specifically, for a typical simulation (P3, Table 2), we computed $\max _{t} \mid V_{B}(t)-$ $V_{B}(0) \mid / V_{B}(0)=4 \%$, where $V_{B}(t)$ denotes the volume of the 'bottom' phase at time $t$, and the maximum is taken over the full duration of the simulation. The mass loss in other simulations was similar. We have not found evidence of any parasitic currents in the simulations. These can arise in levelset methods because of the small-scale smoothing of the viscosity profile and the implementation of the continuum surface force (Meland 


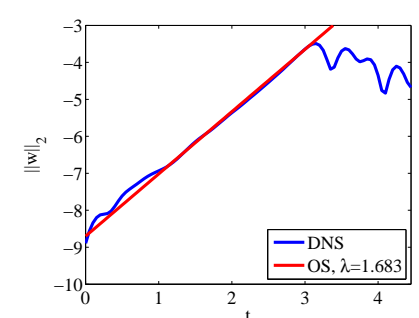

(a) Validation plot: wave growth rate

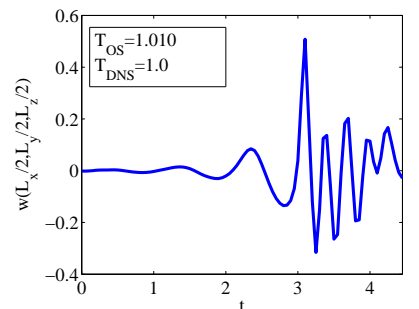

(b) Validation plot: Wave period

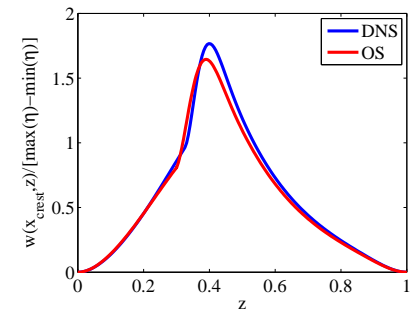

(c) Streamfunction

FIGURE 2. Validation of DNS against separate semi-analytical Orr-Sommerfeld description: (a) shows the $L^{2}$ norm of the perturbation $w$-velocity, and therefore exhibits the wave growth rate; (b) shows the perturbation $w$-velocity at a particular location and therefore demonstrates the wave period; (c) is a plot of the streamfunction normalized as shown in the caption to make contact with OS theory.

et al. 2007); their absence may be anticipated by the large streamwise velocities and rapid instability timescales that are present in the current problem. Comparisons of our results with the quasi-analytic linear theory in Section 2.2 demonstrate that the standard levelset method maintains accuracy with respect to the basic physical model in Equation (2.1). Unless indication is given to the contrary, a grid spacing $\Delta z=1 / 160$ is used throughout this work. This choice is checked on a case-by-case basis using the following tests: first, with $\Delta z=1 / 160$, the full DNS solver reproduces the results of quasi-analytical OrrSommerfeld theory (e.g. Section 2.4). Also, the key nonlinear features (large-amplitude waves, wave overturning, ligaments) are virtually identical for $\Delta z=1 / 160,1 / 225$. Finally, the timestep is chosen such that the advective CFL number based on the maximum basestate velocity $U_{0}(z)$ is at most 0.2 . For the small surface-tension parameters considered throughout this work, timesteps that satisfy the advective CFL criterion automatically satisfy the capillary-wave CFL condition. Note also: although not all the momentum terms are treated in a Crank-Nicolson way (see Equation (2.6)), for the parameters under consideration, the numerical stability of the code was not affected by the diffusive contributions in the momentum equation.

\subsection{Validation of nonlinear DNS code against linear theory}

The code has been rigorously validated with respect to the two-dimensional Orr-Sommerfeld quasi-analytic theory. We have computed the DNS values of the growth rate and wave speed of the most-dangerous temporal mode for the parameter values

$$
(R e, m, \mathcal{S})=(100,30,0.01), \quad h_{0}=0.3
$$

good agreement is obtained with respect to the eigenvalue analysis (Figure 2). The parameters $R e, m$, and $\mathcal{S}$ are varied throughout the work, but the density ratio is maintained at $r=1$ and the film thickness is maintained at $h_{0}=0.3$. The parameter ranges studied are motivated by the work's intended application, namely the modelling of viscous soil removal in plants during cleaning and product turnover operations (Valluri et al. 2010). Here, $\Delta z=1 / 160$ is sufficient for the simulations to have converged, with the convergence criteria as described above in Section 2.3. Also, for Equation (2.7), $\Delta t=10^{-4}$ is sufficient for the advective CFL condition described in Section 2.3 to be satisfied.

As a further test, we examined the inlet/outlet version of the code, with Neumann boundary condition $\phi_{x}(x=0)=\phi_{x}\left(x=L_{x}\right)=0$, subject to an impulsive force (varying only in $x$; no $y$-dependence) applied at very early time, centred at $x=L_{x} / 3$. 


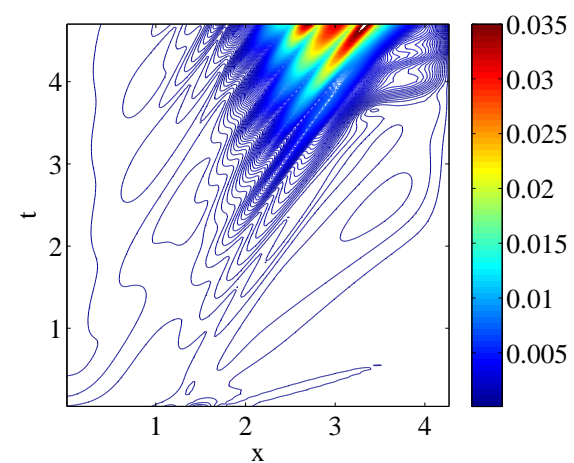

(a)

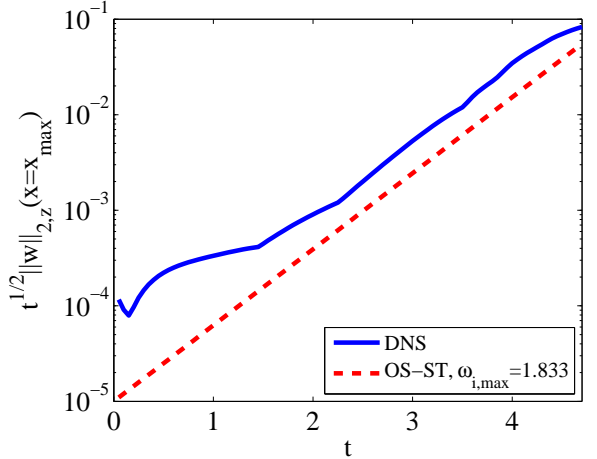

(b)

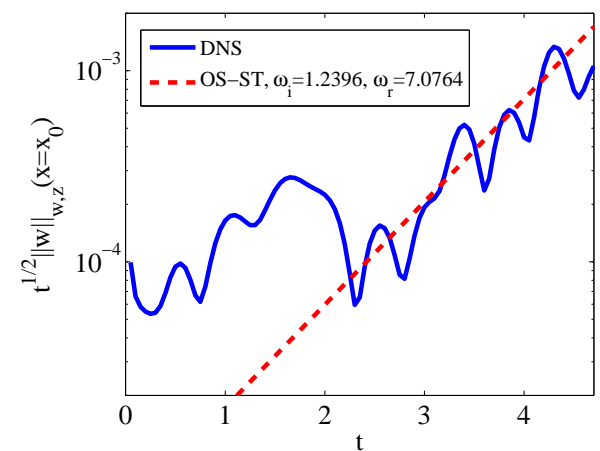

(c)

Figure 3. (a) Space-time plot of the norm $n(x, t)$ for the parameters in Equation (2.7); (b) Comparison between spatio-temporal Orr-Sommerfeld analysis and the DNS at the pulse maximum; (c) Comparison between spatio-temporal Orr-Sommerfeld analysis and the DNS at the source of the disturbance.

We expect standard spatio-temporal Orr-Sommerfeld analysis (Ó Náraigh et al. 2013) to apply to the present case. Thus, we predict that a two-dimensional pulse should form as a result of the imposed initial condition, whose maximum should grow at the same rate as the temporally most-dangerous mode. Moreover, the same Orr-Sommerfeld analysis demonstrates that the flow parameters (2.7) should produce absolute instability, in other words, disturbances grow at the location of the initial impulse, in addition to being convected downstream by the same impulse (Huerre \& Monkewitz 1990; Ó Náraigh et al. 2013). It is therefore also expected that the disturbance should grow at the source, with a growth rate given by the value of the Orr-Sommerfeld frequency $\Omega(\alpha)$ evaluated at the saddle point in the complex $\alpha$-plane. A spacetime plot of the pulse norm

$$
n(x, t)=\left(\int_{0}^{1}|w(x, z, t)|^{2} \mathrm{~d} z\right)^{1 / 2}
$$

is therefore shown in Figure 3(a) ( $w$ represents the perturbation velocity in the wallnormal direction, see Appendix A). Information from this plot is extracted in Figure 3(b), where the pulse maximum is followed. The pulse maximum grows at the same rate as the temporally most-dangerous mode as computed by the Orr-Sommerfeld analysis. Furthermore, the instability grows at the source (absolute instability), and the numerical growth rate is in agreement with the theoretical growth rate computed from the saddle-point of 


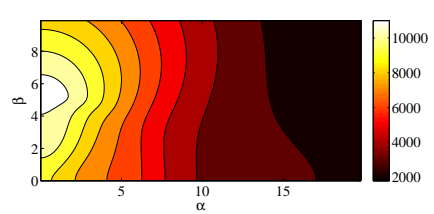

(a) $t=0.02$

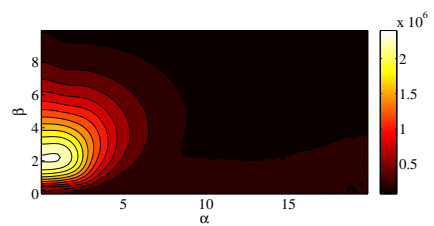

(d) $t=1$

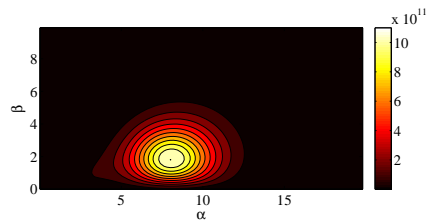

(g) $t=10$

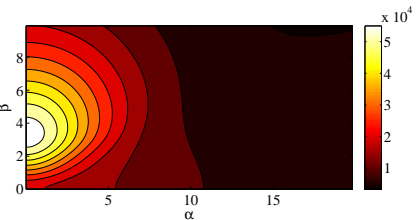

(b) $t=0.05$

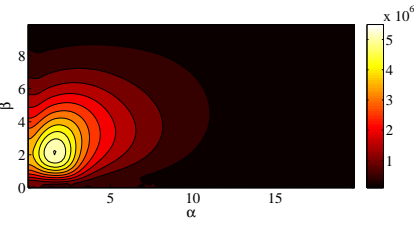

(e) $t=2$

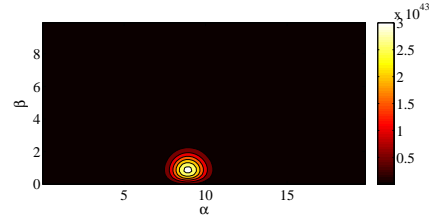

(h) $t=50$

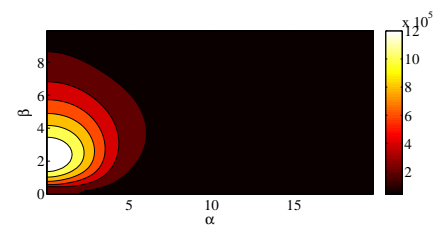

(c) $t=0.5$

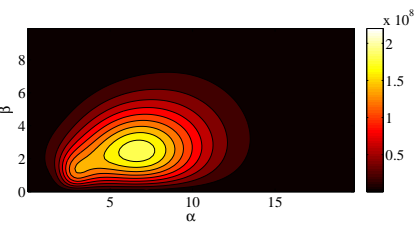

(f) $t=5$

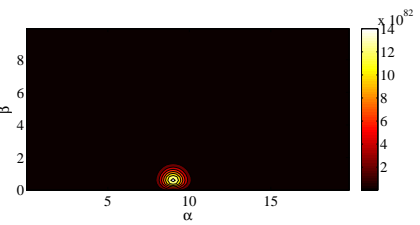

(i) $t=100$

FIGURE 4. Shapshots of the maximum amplification factor $G_{\alpha \beta}(t)$ for transient linear theory, for the parameter values $(m, R e, \mathcal{S})=(30,100,0.01)$.

the complex Orr-Sommerfeld eigenvalue analysis. The measured period in Figure 3(c) is $0.9 \pm 0.1$. The apparent period is only half this amount, because absolute values are taken in plotting $n(x, t)$, rather than a signed velocity. The theoretical value of the period is $2 \pi / 7.095=0.8856$. Thus, the numerical period and the theoretical periods (equivalently wave speeds) agree. The growth rates are also in agreement. A final test involves the study of a parameter class that is convectively unstable. Specifically, we repeated these DNS calculations with $R e=40$ (the other parameters in Equation (2.7) remaining the same). Standard spatio-temporal Orr-Sommerfeld analysis indicates that this parameter class is convectively unstable. This is confirmed by the DNS (not shown): the norm $n(x, t)$ decays at the source location, while the growing disturbance is convected downstream; and grows at a rate corresponding to the temporally most-dangerous mode computed from Orr-Sommerfeld analysis. Further tests concerning the full Orr-Sommerfeld-Squire analysis (not shown here, but demonstrated throughout the paper) demonstrate that the numerical method also captures the three-dimensional modes.

\section{Linear regime - modal and non-modal}

We use the approach based on linear theory outlined in Section 2.2 to study the initial growth of small-amplitude waves, outlining several features of both transient growth and the (asymptotic) dispersion relation for future reference. The calculations are carried out for the parameter values $m=30$, with various values of $\mathcal{S}$ and $R e$.

Figure 4 shows snaphsots of the maximum amplification factor $G_{\alpha \beta}(t)$ at various times, for the parameter set $(m, R e, \mathcal{S})=(30,100,0.01)$. For early times, the purely spanwise modes (i.e. with $\alpha=0$ ) produce the largest transient growth; for later times, the largest amplification occurs for modes that are more streamwise-dominant. This process con- 

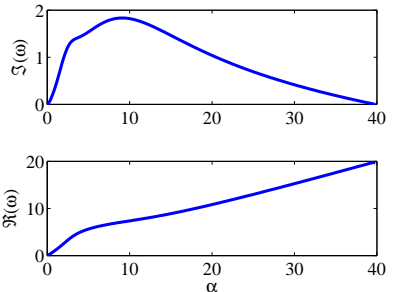

(a) $\beta=0$
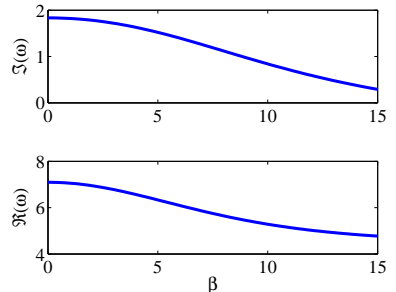

(b) $\alpha=9.0$

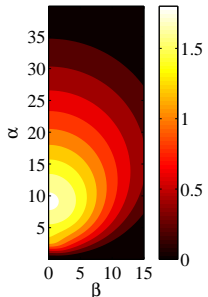

(c)

FIGURE 5. Modal growth rates obtained directly from solving the Orr-Sommerfeld-Squire problem. Here, $(m, R e, \mathcal{S})=(30,100,0.01)$.
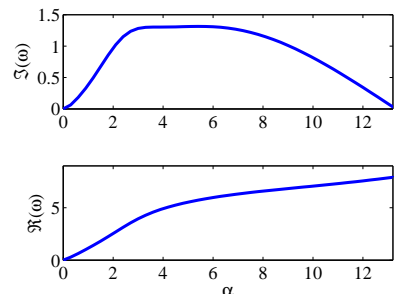

(a) $\beta=0$
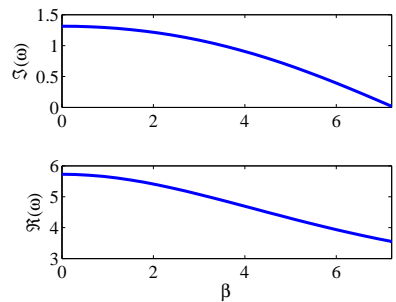

(b) $\alpha=5.4$

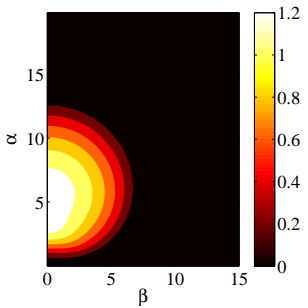

(c)

Figure 6. The same as Figure 5, but with $(m, R e, \mathcal{S})=(30,100,0.1)$.

cludes when the maximum of $G_{\alpha \beta}(t)$ lies on the $\alpha$-axis, but this could not be confirmed due to numerical overflow. However, in support of our claim, we computed the limiting factor

$$
\lim _{\substack{t_{1}, t_{2} \rightarrow \infty \\ t_{2} \gg t_{1}}}\left\{\frac{1}{t_{2}-t_{1}} \log \left[\frac{G_{\alpha \beta}\left(t_{2}\right)}{G_{\alpha \beta}\left(t_{1}\right)}\right]\right\}
$$

and found it to correspond to the asymptotic growth rate computed from eigenvalue Orr-Sommerfeld analysis. This limiting factor is maximal along the $\alpha$-axis. Thus, at late times, maximal growth is centred on the streamwise axis, such that the asymptotic peak of the maximum amplification factor is bound to lie along the same axis. Crucially, most of the transient energy resides at short times in the contribution from the wall-normal vorticity component. This is characteristic of an algebraic instability called the lift-up effect originally observed in single-phase flows (Schmid \& Henningson 2001), but observed also in two-phase mixing layers (Yecko \& Zaleski 2005).

Concerning the asymptotic or eigenvalue analysis of the disturbance, the eigenvalue Orr-Sommerfeld-Squire growth rates for the same parameter set as in Figure 4 are computed and the results are shown in Figure 5. A large range of modes is unstable. The most-dangerous modes are streamwise, and the $\alpha$-dispersion relation in Figure $5(\mathrm{~b})$ is 'flat', such that a band of unstable wavenumbers with very similar growth rates is excited. Upon increasing the surface tension but leaving the other parameters the same, similar features pertain (Figure 6). Here, however, the spanwise growth rates are much smaller than the streamwise ones, and the range of excited modes is narrower. Note also that the $\alpha$-dispersion relation is flatter than before. Consideration is also given to a situation wherein a very narrow range of modes is excited $((m, R e, \mathcal{S})=(30,300,0.3))$. First, the characteristics of the transient growth are very similar to the previous case, with maximum amplification at very early times occurring for the spanwise modes, followed 

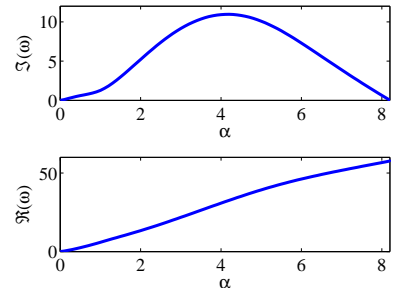

(a) $\beta=0$
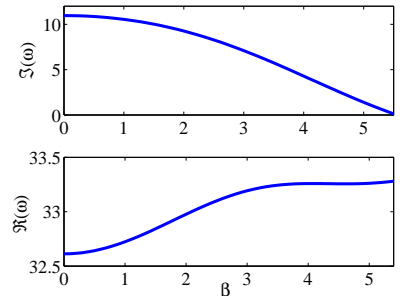

(b) $\alpha=4.2$

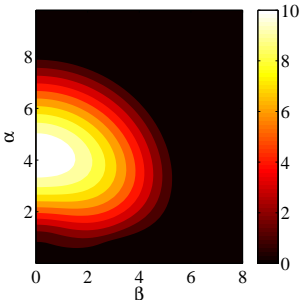

(c)

Figure 7 . The same as Figure 5 , but with $(m, R e, \mathcal{S})=(30,300,0.3)$.

by a shift towards a more streamwise-dominant situation. Concerning the asymptotic results, the dispersion relation valid as $t \rightarrow \infty$ is shown in Figure $7(\mathrm{~b})$. This possesses a sharp peak at $\alpha \approx 4.2$, such that a single mode will feature prominently in the asymptotic evolution of the interface. Also, in spite of the larger Reynolds number than before, three-dimensional waves are more stable in this situation, and only a narrow range of spanwise waves are unstable in the asymptotic limit. These dispersion relations will be used subsequently to explain the linear and weakly nonlinear evolution of the interfacial waves in the full DNS.

\section{Linear and nonlinear waves in periodic simulations}

We begin the presentation of results obtained with direct numerical simulations of cases wherein streamwise-periodic boundary conditions are used. Although these boundary conditions do not reflect the behaviour of a real system, it is appropriate to consider such simulations, for a number of compelling reasons: first, these simulations comprise a 'clean' database, wherein only a small number of modes is active initially, and wherein issues surrounding spatio-temporal growth do not enter, such that the growth of waves can be investigated unambiguously in the framework of linear theory. Also, although a Fourier transform may be taken in a non-periodic domain, the results there are ambiguous because of boundary effects, and such a decomposition is more appropriate in a periodic system.

In these periodic cases, the system is perturbed through the initial condition for the interface location; the initial conditions involve forcing spanwise modes (with a tiny contribution from streamwise modes):

$$
\begin{aligned}
& \eta(x, y, t=0)=h_{0}+\frac{1}{9} A_{0} \sum_{n=1}^{3} \sum_{m=1}^{3} \cos \left(\alpha_{0} n x+\beta_{0} m y+\varphi_{n m}\right) \\
& +\frac{1}{9} \epsilon_{A} \sum_{n=1}^{3} \sum_{m=1}^{3}\left[\begin{array}{c}
\cos \left(n \alpha_{0} x+\varphi_{n m}\right)+\cos \left(m \beta_{0} y+\varphi_{n m}\right) \\
-\cos \left(2 \alpha_{0} n x+\beta_{0} n y+\varphi_{n m}\right)-\cos \left(\alpha_{0} n x+2 \beta_{0} n y+\varphi_{n m}\right)
\end{array}\right],
\end{aligned}
$$

or forcing of both streamwise and spanwise modes:

$$
\begin{aligned}
& \eta(x, y, t=0)=h_{0}+\frac{1}{12} A_{0} \sum_{n=1}^{3} \sum_{m=0}^{3} \cos \left(\alpha_{0} n x+\beta_{0} m y+\varphi_{n m}\right) \\
& +\frac{1}{12} \epsilon_{A} \sum_{n=1}^{3} \sum_{m=0}^{3}\left[\begin{array}{c}
\cos \left(n \alpha_{0} x+\varphi_{n m}\right)+\cos \left(m \beta_{0} y+\varphi_{n m}\right) \\
-\cos \left(2 \alpha_{0} n x+\beta_{0} n y+\varphi_{n m}\right)-\cos \left(\alpha_{0} n x+2 \beta_{0} n y+\varphi_{n m}\right)
\end{array}\right]
\end{aligned}
$$




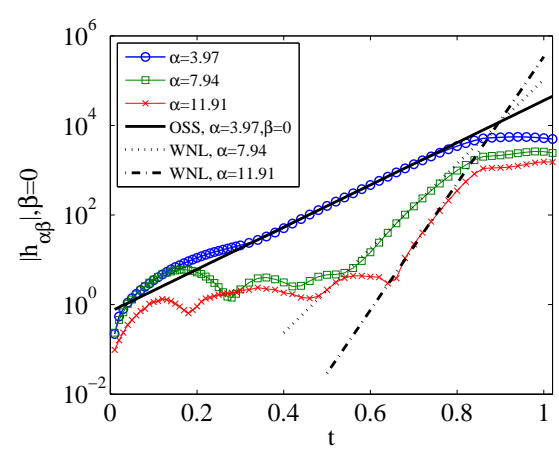

(a)

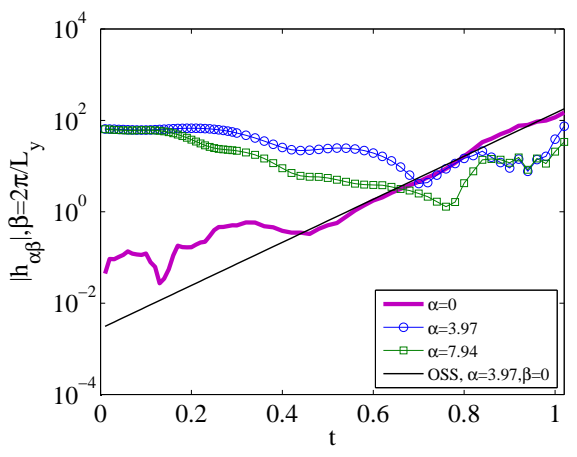

(b)

FiguRE 8. Interfacial spectra for the case P0. (a) Streamwise modes. (b) Spanwise modes, with $\beta=2 \pi / L_{y}$. Both figures show the action of weakly nonlinear mechanisms. In particular in (b) the growth of the purely spanwise mode with $\alpha=0$ is shown.

(note the different limits on the summation index $m$ ). Here, $A_{0}$ is some amplitude, $\varphi_{i j} \in$ $[0,2 \pi)$ is a randomly-selected phase, and $\epsilon_{A}=\left(2.23 \times 10^{-5}\right) A_{0}$ is a small parameter. Also, $\alpha_{0}=2 \pi / L_{x}$ and $\beta_{0}=2 \pi / L_{y}$ are the fundamental wavenumbers in the streamwise and spanwise directions respectively.

\subsection{Subcritical case}

The main focus of this section is on the subcritical case - in this context, by 'subcritical' we mean those parameter regimes that admit linearly unstable streamwise modes but no linearly unstable spanwise modes. Consequently, consideration is given to the simulation P0, involving the parameter set $(m, R e, \mathcal{S})=(30,300,0.3)$. Further geometric parameters are $\left(L_{x}, L_{y}, L_{z}\right)=(1.58,1,1)$, and the initial conditions are those given by Equation (4.1a). The Orr-Sommerfeld-Squire analysis in Section 3 indicates that this parameter set produces instability, with large temporal growth rates for streamwise waves, and no temporal growth for waves with spanwise components. The same quasi-analytical theory predicts that for the geometry prescribed in the P0 simulation, only one unstable mode should be present, with $\alpha=3.97$ and $\Omega_{\mathrm{i}}=10.85$.

The inception of the three-dimensional wave structures in the DNS takes place at early times, when the wave amplitudes are small. To understand this genesis, spectra of the interface height were taken at different times. The spectrum is computed with respect to the interface $\eta(x, y, t)$, defined as follows:

$$
\eta(x, y, t)=\min _{i} Z(x, y, t), \quad Z(x, y, t)=\left\{z_{i} \mid \phi\left(x, y, z_{i}, t\right)=0, i=1,2, \cdots\right\} .
$$

For small-amplitude waves, $i=1$ only, corresponding to a situation wherein there are no overturning waves, droplets, or ligaments. For $i>1$, the interfacial spectrum loses any definite meaning, and spectral data in this regime are not discussed further. The results of the spectral analysis are shown in Figure 8. Figure 8(a) shows a time series of the streamwise modes only. The mode $\alpha=3.96$ grows exponentially fast, at a rate given by OSS theory. As further predicted by the same theory, the other modes do not grow initially. However, as time goes by, the other modes undergo exponential growth, in a manner that is perfectly consistent with weakly nonlinear theory (Barthelet et al. 1995): the $n^{\text {th }}$ multiple of the fundamental wavenumber $\alpha=\alpha_{0}=3.97$ grows at a rate $n \Omega_{\mathrm{i}}\left(\alpha_{0}\right)$.

Consideration is also given to spanwise modes with $\beta=\beta_{0}:=2 \pi / L_{y}$ (Figure $8(\mathrm{~b})$ ), wherein an interesting feature arises: the purely spanwise mode $\left(\alpha=0, \beta_{0}\right)$ that is stable 
in normal-mode linear theory grows exponentially at approximately the same rate as the streamwise most-dangerous mode $(\alpha=3.96, \beta=0)$. It is as if the purely spanwise mode is slaved to the most-dangerous streamwise mode. Further streamwise modes at $(\alpha \neq$ $\left.0, \beta_{0}\right)$ are also excited, but their amplitude remains small compared to the exponentiallygrowing mode until close to the wave turnover time.

These observations are explained in a theoretical framework that couples nonlinear analysis (Craik 1985) to linear transient growth. We start with the following exact nonlinear equations

$$
\left(\mathcal{L}_{\boldsymbol{\alpha}}-\frac{\partial}{\partial t} \mathcal{M}_{\boldsymbol{\alpha}}\right) \chi_{\boldsymbol{\alpha}}=\sum_{\boldsymbol{\beta}} \mathcal{Q}\left[\chi_{\boldsymbol{\beta}}, \chi_{\boldsymbol{\alpha}-\boldsymbol{\beta}}\right]+\sum_{\boldsymbol{\beta}} \sum_{\gamma} \mathcal{R}\left[\chi_{\boldsymbol{\beta}}, \chi_{\boldsymbol{\gamma}}, \chi_{\boldsymbol{\alpha}-\boldsymbol{\beta}-\gamma}\right],
$$

where $\boldsymbol{\alpha}$ denotes a Fourier mode and $\chi_{\boldsymbol{\alpha}}(z, t)$ denotes the complete state of the system in the same Fourier mode (i.e. $\chi$ contains information about velocity, vorticity, and interface height, as in the Orr-Sommerfeld-Squire (OSS) theory) (Schmid \& Henningson 2001; Craik 1985; King \& McCready 2000). Each normal mode $\boldsymbol{\alpha}$ with corresponding state $\chi_{\boldsymbol{\alpha}}$ can be decopmposed further into a sum over OSS eigenfunctions:

$$
\chi_{\boldsymbol{\alpha}}=\sum_{m=1}^{\infty} A_{\boldsymbol{\alpha}}^{(m)}(t) v_{\boldsymbol{\alpha}}^{(m)}(z), \quad\left(\mathcal{L}_{\boldsymbol{\alpha}}-\lambda_{\boldsymbol{\alpha}}^{(m)} \mathcal{M}_{\boldsymbol{\alpha}}\right) v_{\boldsymbol{\alpha}}^{(m)}=0 .
$$

This gives rise to the following infinite set of amplitude equations:

$$
\left(\frac{\mathrm{d}}{\mathrm{d} t}-\lambda_{\boldsymbol{\alpha}}^{(m)}\right) A_{\boldsymbol{\alpha}}^{(m)}=\sum_{\boldsymbol{\beta}} \sum_{p, q=1}^{\infty} \mu_{\boldsymbol{\alpha}, \boldsymbol{\beta}}^{(m, p, q)} A_{\boldsymbol{\beta}}^{(p)} A_{\boldsymbol{\alpha}-\boldsymbol{\beta}}^{(q)}+\sum_{\boldsymbol{\beta}} \sum_{\boldsymbol{\gamma}} \sum_{p, q, r=1}^{\infty} \nu_{\boldsymbol{\alpha}, \boldsymbol{\beta}, \boldsymbol{\gamma}}^{(m, p, q)} A_{\boldsymbol{\beta}}^{(p)} A_{\boldsymbol{\gamma}}^{(q)} A_{\boldsymbol{\alpha}-\boldsymbol{\beta}-\boldsymbol{\gamma}}^{(r)}
$$

Equally, the right-hand side of the basic equation (4.3) can be decomposed as follows:

$$
\begin{aligned}
\left(\mathcal{L}_{\boldsymbol{\alpha}}-\frac{\partial}{\partial t} M_{\boldsymbol{\alpha}}\right) \chi_{\boldsymbol{\alpha}}= & \sum_{\boldsymbol{\beta}} \mathcal{Q}\left[\sum_{p=1}^{\infty} A_{\boldsymbol{\beta}}^{(p)} v_{\boldsymbol{\beta}}^{(p)}, \sum_{q=1}^{\infty} A_{\boldsymbol{\beta}}^{(q)} v_{\boldsymbol{\alpha}-\boldsymbol{\beta}}^{(q)}\right] \\
& +\sum_{\boldsymbol{\beta}} \sum_{\boldsymbol{\gamma}} \mathcal{R}\left[\sum_{p=1}^{\infty} A_{\boldsymbol{\beta}}^{(p)} v_{\boldsymbol{\beta}}^{(p)}, \sum_{q=1}^{\infty} A_{\gamma}^{(q)} v_{\boldsymbol{\beta}}^{(q)}, \sum_{r=1}^{\infty} A_{\boldsymbol{\beta}}^{(r)} v_{\boldsymbol{\alpha}-\boldsymbol{\beta}-\boldsymbol{\gamma}}^{(r)}\right]
\end{aligned}
$$

Based on the results of the simulation P0, we carry out simplifications of Equation (4.5) to extract a phenomenological theory from the same equation. The situation is complicated, and different families of modes exhibit different types of nonlinear behaviour. We address each mode family in turn.

\section{Purely streamwise modes}

For the simulation under consideration, standard OSS eigenvalue analysis shows that the mode

$$
\boldsymbol{\alpha}=\boldsymbol{\alpha}_{0}:=\left(\frac{2 \pi}{L_{x}}, 0\right)
$$

grows rapidly according to linear theory, while all other modes are linearly stable (the mode $\boldsymbol{\alpha}_{0}$ is the most-dangerous mode). Indeed, the exponential growth of the mode $\boldsymbol{\alpha}_{0}$ holds up until the breaking point of the nonlinear waves. In addition, the 'harmonics', i.e. the modes $\boldsymbol{\alpha}=\left(\left(2 \pi / L_{x}\right) n, 0\right)$, with $n=2,3, \cdots$ appear in the simulation to be 'slaved' to the most-dangerous mode. This behaviour of the growth rates in these instances has already been alluded to in Figure 8(a). Furthermore, the behaviour of the corresponding wave frequencies is shown in Figure 9 and the results summarized in Table 1. These results 


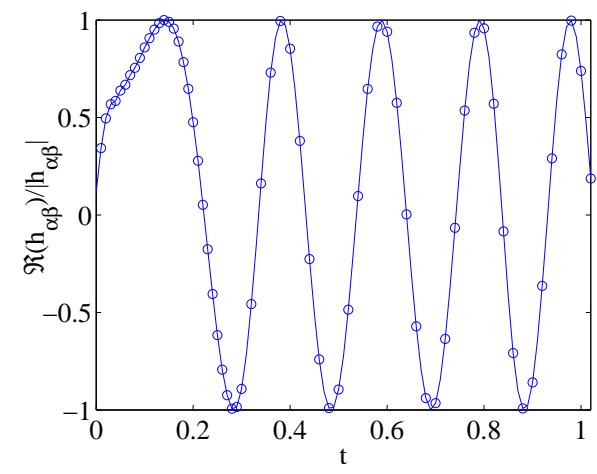

(a)

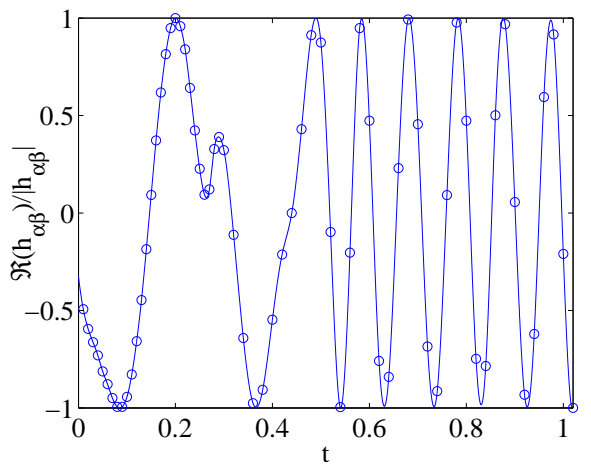

(b)

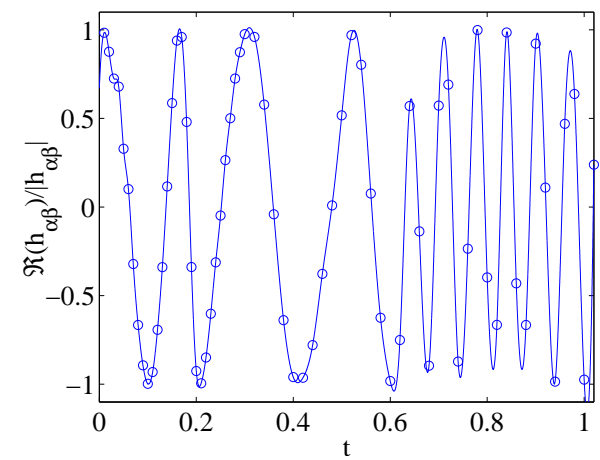

(c)

Figure 9. Plot of $h_{\boldsymbol{\alpha}}(t) /\left|h_{\boldsymbol{\alpha}}(t)\right|$ for (a) the most-dangerous mode; (b) the first harmonic; (c) the second harmonic. Here $h_{\boldsymbol{\alpha}}(t)$ denotes the two-dimensional Fourier transform of the interfacial height $h(x, y, t)$, at time $t$, and at wavenumber $\boldsymbol{\alpha}$.

\begin{tabular}{|c|c|c|c|c|}
\hline$\alpha$ & \multicolumn{4}{|c|}{$|n| n \Omega_{\mathrm{r}, O S}\left(\alpha_{0}, 0\right)\left|\Omega_{D N S}\right|$ Sample time } \\
\hline 3.97 & 1 & 30.4 & 29.9 & $t>0.48$ \\
\hline 7.94 & 2 & 60.8 & 60.6 & $t>0.54$ \\
\hline 11.01 & 3 & 91.2 & 92.8 & $t>0.68$ \\
\hline
\end{tabular}

TABLE 1. Dominant wave frequency at intermediate times: comparison between DNS and Stuart-Landau / Slow-Manifold theory. Here $n$ labels the harmonic: $\alpha_{0}=2 \pi / L_{x}$ is the fundamental wavenumber, and the considered wavenumbers are in the relation $\alpha=n \alpha_{0}$.

show that at early times, a range of frequencies is present, consistent with the fact that each normal mode $\chi_{\boldsymbol{\alpha}}(z, t)$ contains a mixture of OSS modes (hence frequencies). At late times, a single frequency becomes apparent, whose value is given to within a small measurement error by the relation

$$
\Omega_{\mathrm{r}, D N S}\left(n \alpha_{0}, 0\right)=n \Omega_{\mathrm{r}, O S}\left(\alpha_{0}, 0\right), \quad n=1,2, \cdots
$$


These results (once the transient effects dissipate) are entirely consistent with SlowManifold theory applicable to a handful of modes. In particular, it suffices to consider only one OSS mode per Fourier mode, such that the amplitude equations (4.4) at quadratic order reduce to the following form:

$$
\begin{aligned}
\frac{d A_{\left(\alpha_{0}, 0\right)}}{d t} & =\lambda_{\left(\alpha_{0}, 0\right)} A_{\left(\alpha_{0}, 0\right)} \\
\frac{d A_{\left(2 \alpha_{0}, 0\right)}}{d t} & =\lambda_{\left(2 \alpha_{0}, 0\right)} A_{\left(2 \alpha_{0}, 0\right)}+\mu_{\left(\alpha_{0}, 0\right),\left(2 \alpha_{0}, 0\right)} A_{\left(\alpha_{0}, 0\right)}^{2} \\
\frac{d A_{\left(3 \alpha_{0}, 0\right)}}{d t} & =\lambda_{\left(3 \alpha_{0}, 0\right)} A_{\left(3 \alpha_{0}, 0\right)}+\left[\mu_{\left(\alpha_{0}, 0\right),\left(3 \alpha_{0}, 0\right)}+\mu_{\left(2 \alpha_{0}, 0\right),\left(3 \alpha_{0}, \beta_{0}\right)}\right] A_{\left(\alpha_{0}, 0\right)} A_{\left(2 \alpha_{0}, 0\right)},(4.6)
\end{aligned}
$$

where all superscripts take the value $m=1$ and are suppressed for brevity. Given the separation of scales inherent in this particular problem:

$$
\Re\left(\lambda_{\left(\alpha_{0}, 0\right)}\right) \gg 1, \quad \forall \boldsymbol{\alpha} \neq\left(\alpha_{0}, 0\right), \Re\left(\lambda_{\boldsymbol{\alpha}}\right) \ll-1,
$$

Slow-Manifold theory applies (Pavliotis \& Stuart 2008), such that

$$
A_{n \alpha_{0}, 0} \propto A_{\alpha_{0}, 0}^{n},
$$

thereby explaining the results observed in the DNS. The behaviour described in Equation (4.7) has been observed in the same simulation for $n=4,5$ too. Note also that the same conclusion as Equation (4.7) is reached even when cubic-order interactions are taken into account (Barthelet et al. 1995; King \& McCready 2000). These facts confirm the robustness of the match between theory and simulation for this particular (streamwise) mode family.

Note: using rigorous homogenization theory (Pavliotis \& Stuart 2008), one can show that a dynamical system does not need to be close to 'criticality' in order for slaving to occur (in this context, by 'criticality' we mean that a single mode is barely linearly unstable, and all other modes are linearly stable). Proximity to criticality is sufficient for slaving to occur, but is not necessary. Instead, the necessary condition for slaving to occur is for a separation of scales should exist: the natural evolution timescale of (say) $A_{\left(\alpha_{0}, 0\right)}$ should be well separated from the timescales of the other Fourier modes in the problem, as is the case in the present discussion.

$$
\text { The spanwise mode }\left(\alpha_{0}, \beta_{0}\right)
$$

In the P0 simulation, linear theory applies to this mode until the breaking point. Care is needed here: the measured Fourier mode contains a mixture of OSS modes, such that the temporal evolution is not a simple exponential decay characterized by a single damping rate: a number of damping rates and oscillation frequencies apply. This description is confirmed unambigously by using our own independent linearized DNS (L-DNS) technique (Ó Náraigh et al. 2013) and comparing the results with those arising from the DNS using the TPLS solver. The results of this comparison are shown in Figure 10(a)-(b). A spectral analysis of the temporal evolution associated with the L-DNS is shown in Figure 10(c). Two sharp peaks are on display, corresponding to two stable OSS eigenmodes. Clearly, the behaviour of this particular Fourier mode is explained adequately with straightforward linear theory, albeit that a combination of OSS eigenmodes is active.

$$
\text { The purely spanwise mode }\left(0, \beta_{0}\right)
$$

In the simulation, this mode is observed to grow exponentially at a rate close to that of the most-dangerous mode. Additionally, the frequency of modulation is low (Figure 11). 


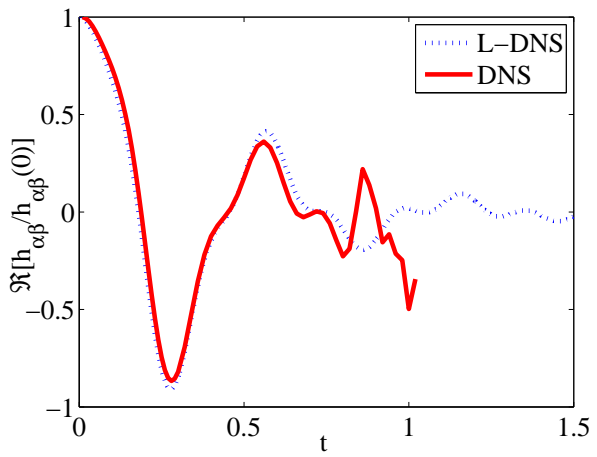

(a)

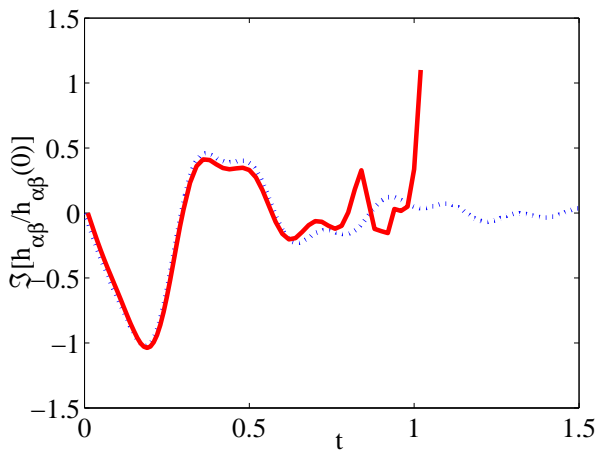

(b)

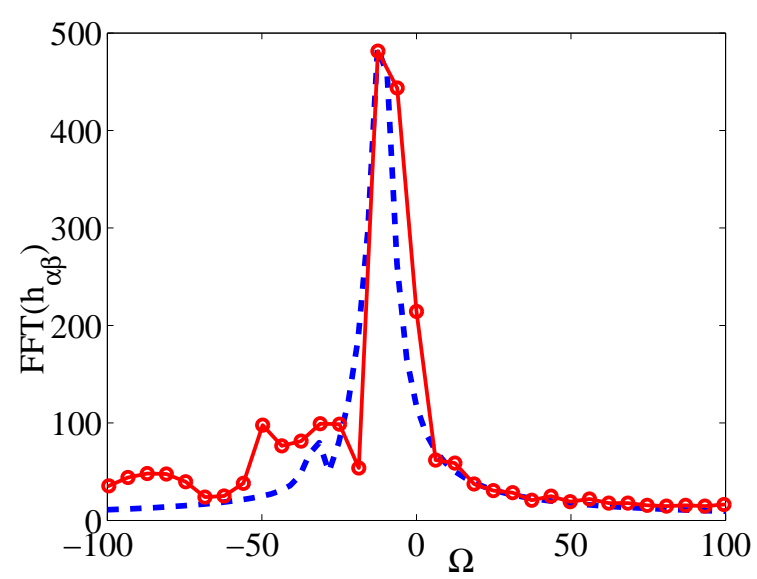

(c)

Figure 10. Dynamics of the spanwise Fourier mode $\boldsymbol{\alpha}=\left(\alpha_{0}, \beta_{0}\right)$. (a) Real part of $h_{\boldsymbol{\alpha}}(t) / h_{\boldsymbol{\alpha}}(0)$ with a comparison between the full DNS and the linearized DNS; (b) imaginary part of the same; (c) Spectral analysis (frequency space) based on the linearized DNS (broken line) and the full nonlinear DNS (solid line, with dots, spectrum taken between $t=0$ and $t=1.02$ ). The two most-prominent peaks in the L-DNS in fact coincide with the OSS modal frequencies.

These two observations are not explained either by linear theory, or by a naive application of Slow-Manifold theory. However, a more refined version of the latter can be invoked, as we now explain.

We start with the basic equation (4.5) truncated at quadratic order. We take $\boldsymbol{\alpha}=$ $\left(0, \beta_{0}\right)$, where $\beta_{0}=2 \pi / L_{y}$. With regard to the summation variable $\boldsymbol{\beta}$, in a first approximation, only those values of $\boldsymbol{\beta}$ and $\boldsymbol{\alpha}-\boldsymbol{\beta}$ that induce a coupling to the most-dangerous mode matter. Consequently, one obtains the following reduction in the complexity, for the mode $\boldsymbol{\alpha}=\left(0, \beta_{0}\right)$ :

$$
\left(\mathcal{L}_{\boldsymbol{\alpha}}-\frac{\partial}{\partial t} \mathcal{M}_{\boldsymbol{\alpha}}\right) \chi_{\boldsymbol{\alpha}}=\mathcal{Q}\left[A_{\left(\alpha_{0}, 0\right)}^{(1)} v_{\left(\alpha_{0}, 0\right)}^{(1)}, \sum_{q=1}^{\infty} A_{\left(-\alpha_{0}, \beta_{0}\right)}^{(q)} v_{\left(-\alpha_{0}, \beta_{0}\right)}^{(q)}\right]
$$

For the present simulation, the Fourier mode $\left(-\alpha_{0}, \beta_{0}\right)$ is not excited directly by the initial condition (but it will of course appear as the simulation progresses, due to nonlinear coupling). Consequently, it can be neglected in a lowest-order approximation, and 


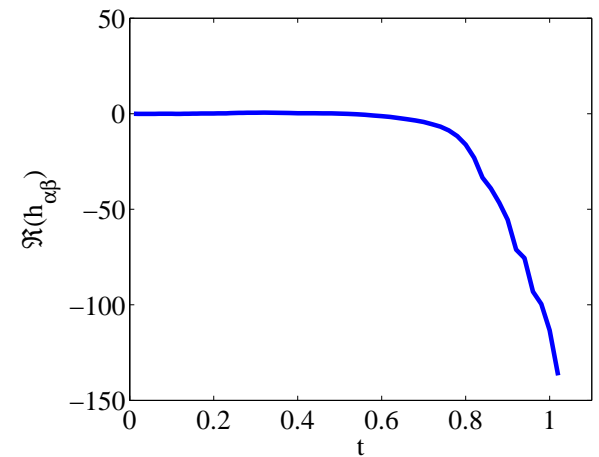

(a)

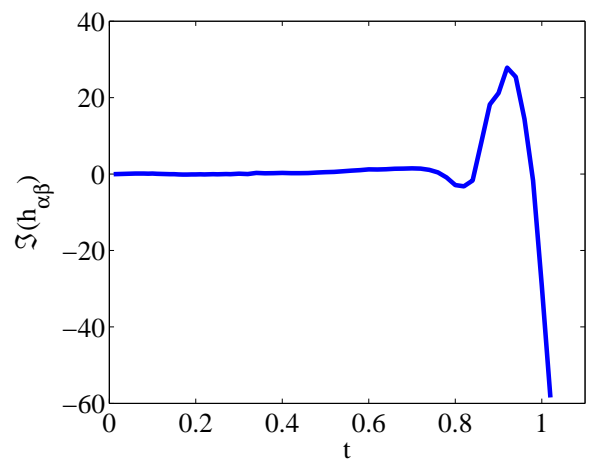

(b)

FigURE 11. Dynamics of the purely spanwise Fourier mode $\left(0, \beta_{0}\right)$, with evidence of evolution over a dominant long timescale $T>1, \Omega<2 \pi$.

Equation (4.8) is reduced further to the following model equation:

$$
\left(\mathcal{L}_{\boldsymbol{\alpha}}-\frac{\partial}{\partial t} \mathcal{M}_{\boldsymbol{\alpha}}\right) \chi_{\boldsymbol{\alpha}}=\mathcal{Q}\left[A_{\left(-\alpha_{0}, 0\right)}^{(1)} v_{\left(\alpha_{0}, 0\right)}^{(1)}, \sum_{q=1}^{\infty} A_{\left(\alpha_{0}, \beta_{0}\right)}^{(q)} v_{\left(\alpha_{0}, \beta_{0}\right)}^{(q)}\right]
$$

Finally, from the DNS and from the previous discussion, the Fourier and OSS modes appearing on the right-hand side obey linear theory. Also, the quadratic interaction function $\mathcal{Q}(\cdot, \cdot)$ is bilinear, meaning that Equation (4.10) now simplifies in the following manner:

$$
\begin{aligned}
\left(L_{\boldsymbol{\alpha}}-\frac{\partial}{\partial t} M_{\boldsymbol{\alpha}}\right) \chi_{\boldsymbol{\alpha}} & =\sum_{q=1}^{\infty} \exp \left[\left(\overline{\lambda_{\alpha_{0}, 0}^{(1)}}+\lambda_{\alpha_{0}, \beta_{0}}^{(q)}\right) t\right] \mathcal{Q}\left[A_{\left(-\alpha_{0}, 0\right)}^{(1)}(0) v_{\left(\alpha_{0}, 0\right)}^{(1)}, A_{\left(\alpha_{0}, \beta_{0}\right)}^{(q)}(0) v_{\left(\alpha_{0}, \beta_{0}\right)}^{(q)}\right] \\
& \left.:=\sum_{q=1}^{\infty} F_{q}\left(z ; \alpha_{0}, \beta_{0}\right) \exp \left[\overline{\left(\lambda_{\alpha_{0}, 0}^{(1)}\right.}+\lambda_{\alpha_{0}, \beta_{0}}^{(q)}\right) t\right]
\end{aligned}
$$

Equation (4.10) is a forced linear PDE, where the forcing contains a number of amplification factors and a number of complex frequencies in the exponential form $\mathrm{e}^{\lambda_{\text {eff }} t}$, where

$$
\left\{\lambda_{\text {eff }} \in \mathbb{C} \mid \lambda_{\text {eff }}=\overline{\lambda_{\alpha_{0}, 0}^{(1)}}+\lambda_{\left(\alpha_{0}, \beta_{0}\right)}^{(q)}, q=1,2 \cdots\right\} .
$$

Given that the OSS eigenvalues for the confined flow considered herein form a discrete set, the possibility that $\lambda_{\text {eff }}^{(q)}$ will coincide with an eigenvalue of the OSS operator at the Fourier mode $\left(0, \beta_{0}\right)$ is anomolous, and need not be considered here. Therefore, assuming non-coincidence, the equation system (4.10) will select that particular value of $\lambda_{\text {eff }}^{(q)}$ that maximizes the resolvent norm (Schmid \& Henningson 2001)

$$
R(z)=\left\|\left(\mathcal{L}_{\boldsymbol{\alpha}}-z \mathcal{M}_{\boldsymbol{\alpha}}\right)^{-1}\right\|
$$

We call this phenomenon 'quasi-resonance' (the term 'resonance' is restricted to mean $R(z)=\infty$, i.e. a $z$-value correspoding to an eigenvalue of the linear problem at $\left.\left(0, \beta_{0}\right)\right)$. We have plotted the resolvent norm as a function of the variable $z$, with $z$ ranging over (a portion of) the complex plane. The results are shown in Figure 12. We have also plotted the resolvent norm evaluated at those particular $z$-values that correspond to eigenvalue combinations $\overline{\lambda_{\alpha_{0}, 0}^{(1)}}+\lambda_{\left(\alpha_{0}, \beta_{0}\right)}^{(q)}$. The eigenvalue combination that maximizes the resolvent 


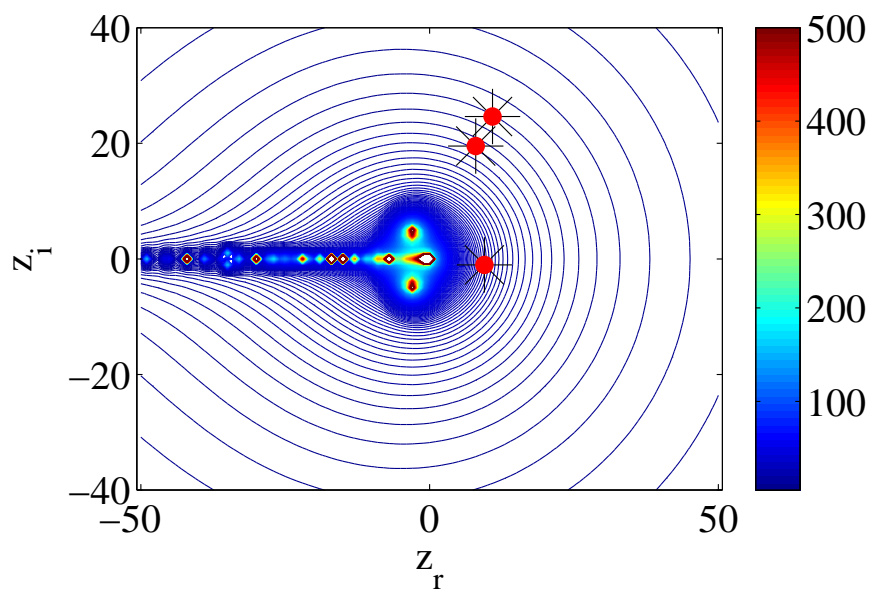

Figure 12. The resolvent norm $R(z)$ for the case $\boldsymbol{\alpha}=\left(0, \beta_{0}\right)$, for a range of complex $z$-values. Poles occur at $z$-values corresponding to the OSS eigenvalues ('resonance'). The resolvent norm $R(z)$ with $z$ restricted to the set of frequencies in Equation (4.11) is a discrete set. The stars in the figure show the three largest values attained by $R(z)$ when one restricts to these discrete values.

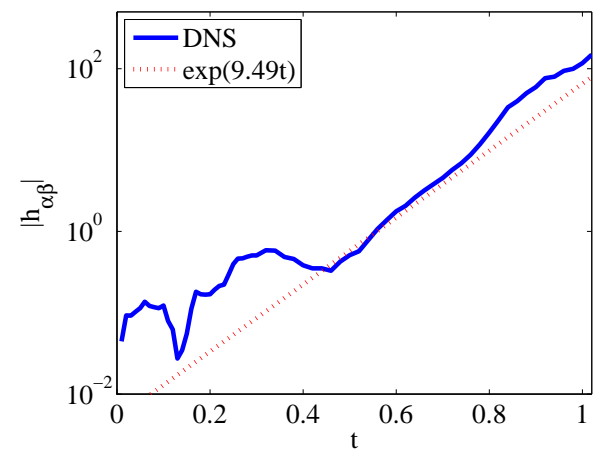

(a)

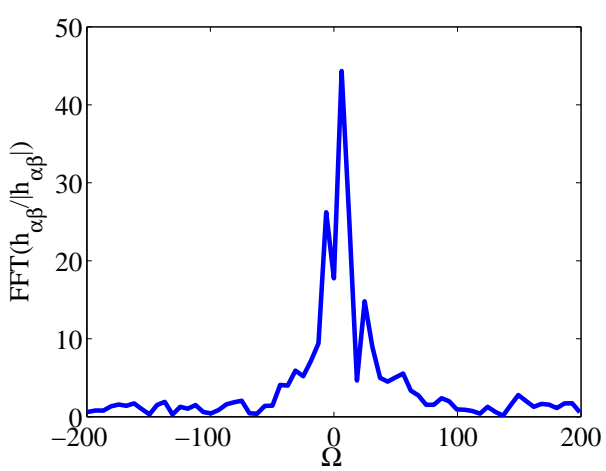

(b)

Figure 13. (a) Growth of purely spanwise Fourier mode $\boldsymbol{\alpha}=\left(0, \beta_{0}\right)$ with comparison to quasilinear / weakly nonlinear theory as described in this section; (b) Spectral analysis of the same Fourier mode; the spectrum is taken between $t=0$ and $t=1.02$

norm is found to be

$$
\lambda_{\text {eff }}=\overline{\lambda_{\left(\alpha_{0}, 0\right)}}+(-1.38-30.4 \mathrm{i})=(10.91+30.62 \mathrm{i})+(-1.416-31.92 \mathrm{i})=9.494-1.300 \mathrm{i}
$$

The exponential growth exhibted in the DNS is compared against this predicted growth rate in Figure 13(a). Good agreement is obtained between the quasilinear / weakly nonlinear analysis proposed in this section and the full DNS. A spectral analysis of the same mode is shown in Figure 13(b), based here on the full DNS. The Fourier transform (in frequency space) is taken with respect to $h_{(\alpha, \beta)} /\left|h_{(\alpha, \beta)}\right|$ to reduce the influence of the growth rates on the frequency characterization. In view of the short window of time over which spectral data are available (i.e. before wave overturning, and short compared to 


\begin{tabular}{|c|c|c|c|}
\hline \multicolumn{2}{|c|}{ Case|Parameters } & \multirow{2}{*}{$\begin{array}{l}\text { | Geometric Parameters } \\
\mid\left(L_{x}, L_{y}, L_{z}\right)=(2,1,1)\end{array}$} & \multirow[t]{2}{*}{ | Figure } \\
\hline $\mathrm{P} 1$ & $\begin{array}{l}A_{0}=0.002, \mathcal{S}=0.01, R e=100, \text { ICs: Equa- } \\
\text { tion }(4.1 a)\end{array}$ & & \\
\hline $\mathrm{P} 2$ & $\begin{array}{l}A_{0}=0.002, \mathcal{S}=0.01, R e=100, \text { ICs: Equa- } \\
\text { tion }(4.1 b)\end{array}$ & $\left(L_{x}, L_{y}, L_{z}\right)=(2,1,1)$ & Figure 14 \\
\hline $\mathrm{P} 3$ & $\begin{array}{l}A_{0}=0.02, \quad \mathcal{S}=0.1, \quad R e=100, \text { ICs: Equa- } \\
\text { tion }(4.1 a)\end{array}$ & $\left(L_{x}, L_{y}, L_{z}\right)=(2,1,1)$ & \\
\hline
\end{tabular}

TABLE 2. Summary of those periodic simulations involving a number of linearly unstable modes, with $m=30$.

the dominant long timescale), quantitative comparison is difficult. However, the results are consistent with the weakly nonlinear analysis: the most-prominent frequency is measured at $\Omega= \pm(6 \pm 3)$, consistent with the data in Figure 11 and the 'quasi-resonance' in Equation (4.12). A second peak at $\Omega=24 \pm 3$ is consistent with further 'quasi-resonance' corresponding to the third-largest value of the resolvent norm in the frequency set (4.11).

\subsection{Supercritical route to three-dimensional waves}

We also consider the route to ligament formation in periodic domains for supercritical parameter sets, such that both streamwise and spanwise modes are linearly unstable. Although it may be expected that in the linear regime, the initial growth of the waves should be governed by standard modal linear instability, the subsequent behaviour is also investigated, including the relevance of the weakly non-linear theory set out in the previous section for the subcritical case.

A typical resulting flow behaviour is illustrated in Figure 14 for the case P2 (defined in Table 2). In this case, under linear theory, two-dimensional and three-dimensional waves are strongly amplified, with growth rates that are comparable in magnitude (e.g. Figure 5); in particular,

$$
\frac{\Omega_{\mathrm{i}}(\alpha=9.42, \beta=0)}{\Omega_{\mathrm{i}}\left(\alpha=9.42, \beta=2 \pi / L_{y}\right)}=\frac{1.832}{1.364} \approx 1.343,
$$

from the same Orr-Sommerfeld-Squire analysis. We have investigated the physical mechanism at work during this early-stage growth. For the cases P1-P3 (See Table 2), we analysed the tangential-stress distribution at the interface, and the results from the DNS are consistent the standard conclusion from linear theory that the instability is precipitated by a mismatch in the viscosity across the interface (e.g. Yih (1967); Boomkamp \& Miesen (1996)). This was confirmed using an energy budget carried out in the framework of linear theory. The case P0 exhibits a similar tangential-stress distribution, except that a subsequent energy budget based again on linear theory revealed that other contributions to the perturbation energy are dominant, with the Reynolds-stress term in the upper layer being the largest contributor. This result indicates that the character of the most-dangerous mode changes upon inceasing the Reynolds number, such that at higher Reynolds number, a more shear-like mode emerges. Note, however, because several terms give a positive contribution to the kinetic energy supplied to the perturbations, the distinction between the mode types is somewhat blurred (Boomkamp \& Miesen 1996). 


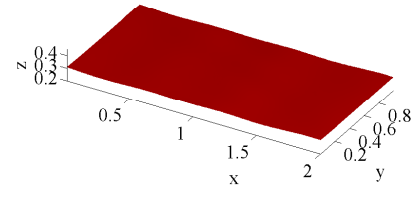

(a) $t=1.5$

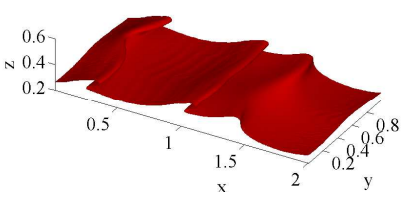

(d) $t=3.5$

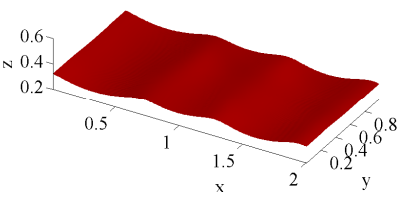

(b) $t=2.5$

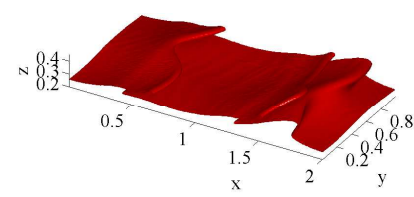

(e) $t=3.8$

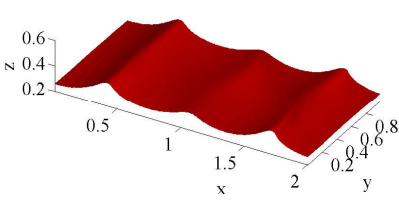

(c) $t=3.0$

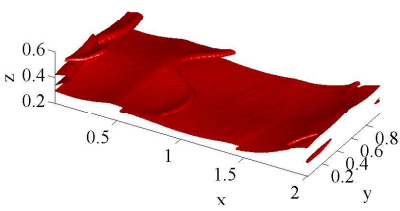

(f) $t=4.0$

Figure 14. DNS solution for case P2 $\left(\mathcal{S}=0.01, A_{0}=0.002\right.$, and initial conditions given by Equation $(4.1 b))$. Snapshots of the interface profile at various times, coloured by wave elevation.

A quantitative analysis of the results has been conducted in terms of interfacial spectra for the P2 simulation (Figure 14). It is clear from this figure that the streamwise and spanwise waves that develop on the interface in the snapshots arise initially directly from linear theory: the streamwise and spanwise 'long' waves with $\alpha=3.14,6.28,9.42$ in Figure 15 enjoy a period of exponential growth at a rate given by Orr-Sommerfeld-Squire theory (shorter waves exhibit weak nonlinearity and are discussed below). A comparison between the interfacial snapshots and the spectral plots in Figure 15 indicates that this linear regime persists here until close to the point of overturning, similar to observations in prior studies for related two-dimensional flows (Valluri et al. 2007, 2010). Note that in Figure 15, 'kinks' in the time series of the spectral amplitudes signal wave overturning, and roughly coincide with the end of the regime of exponential growth in the amplitudes (i.e. the end of the regime of linearized dynamics).

Although linear theory persists until very close to the point of wave overturning, the non-linear overturning itself is a key stage. Also, it is of interest to understand the discrepancy in Figure 15, wherein the relatively short wave at $\alpha=12.56$ grows at a faster rate than that predicted by linear theory. We study the nonlinear aspects of the flow behaviour in terms of snapshots of the interface spectra, at relatively late times, focusing on streamwise modes (Figure 16). The simplified weakly non-linear theory in the work by Barthelet et al. (1995) is not expected to pertain: not only overtones of the linearly most-dangerous mode, but also other combinations are present, due to the fact that the parameter regime under investigation is far beyond criticality. Indeed, the most prominent wave to be excited in the weakly non-linear regime is at $\alpha=15.70$, which suggests a three-wave interaction involving the linearly-excited modes at $\alpha=6.28$ and $\alpha=9.42$. The second most-prominent wave to be excited in the non-linear regime is at $\alpha=18.84$, corresponding to an overtone of the linearly most-dangerous mode at $\alpha=9.42$; the overtone thus contributes to the steepening of the $\alpha=9.42$ wave. In this way, the relatively short waves in the simulation are amplified at rates above those implied by 


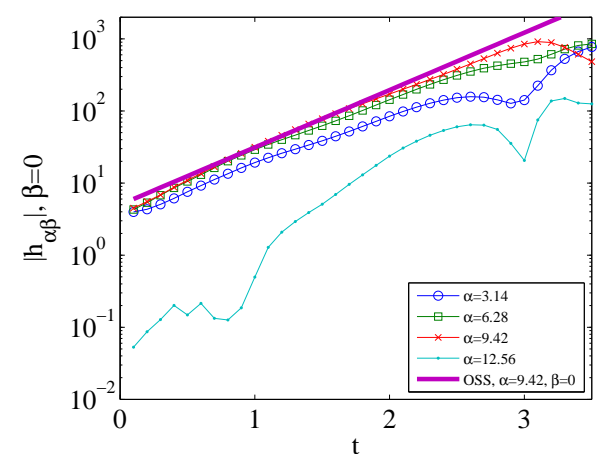

(a) P2, streamwise modes

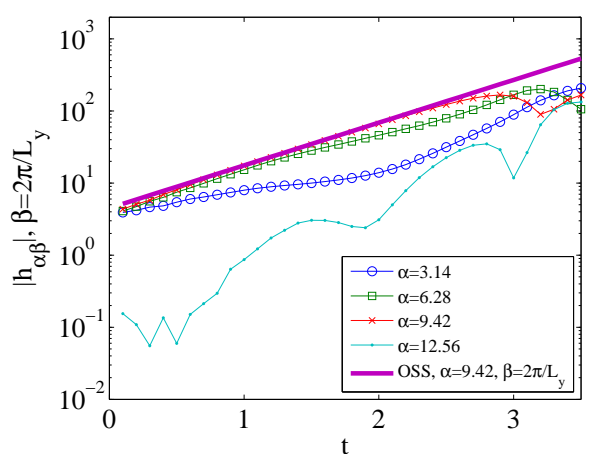

(b) P2, spanwise modes

FIGURE 15. Interfacial spectra for the cases P2, correspnding to initial conditions with an equal mixture of spanwise and streamwise modes, with and $A_{0}=0.002$. Here $(R e, m, \mathcal{S})=(100,30,0.01)$, The downwards-pointing kinks coincide with wave overturning.

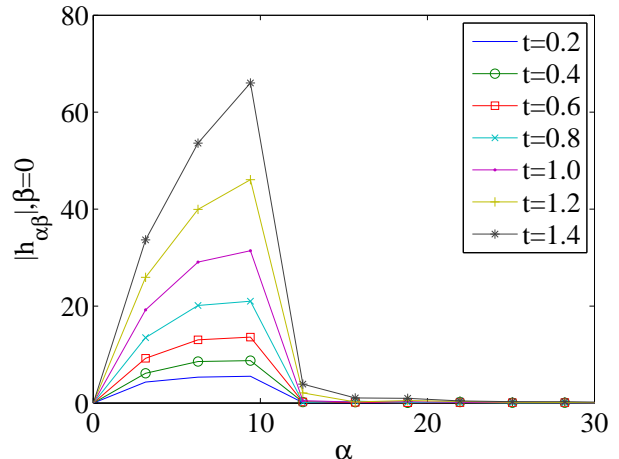

(a)

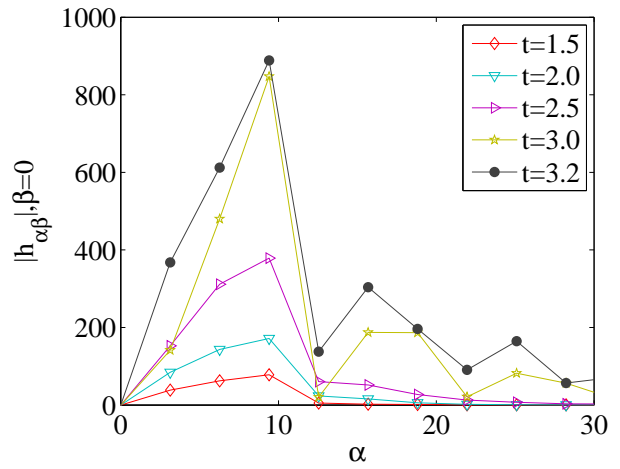

(b)

FIGURE 16. Snapshots of the interfacial spectra (streamwise modes) for the case P2. (a) Linear regime; (b) weakly non-linear regime.

linear theory. This result can also be used to explain why the relatively short wave at $\alpha=12.56$ does not obey linear theory, whereas longer waves $(\alpha=3.14,6.28,9.42)$ do. We also investigated a further simulation along exactly the same lines, namely P1 (Table 2), corresponding to the same parameter set as P2, but with initial conditions that include only a tiny contribution from streamwise modes. The results are very similar to the case P2, highlighting the importance of the intrnisic linear-instability mechanism which is at work in an identical manner in the two simulations P1 and P2.

Finally, we examine the robustness of the weakly nonlinear theory for spanwise modes developed in the subcritical context (e.g. Equations (4.3)). Although the discussion around Equation (4.3) takes place in the context of a simulation wherein only a single streamwise mode is linearly unstable, the derivation of Equation (4.10) will carry over in an approximate sense to the cases P1-P3. For, only a number of unstable modes fit inside the periodic channels. In the simulations P1-P3, the dispersion relations are 'flat', meaning that all unstable modes have a growth rate comparable to the mostdangerous modal growth rate. The spanwise (linearly stable) mode will therefore 'see' the streamwise modes as a single unstable mode, and under the coupling described in 


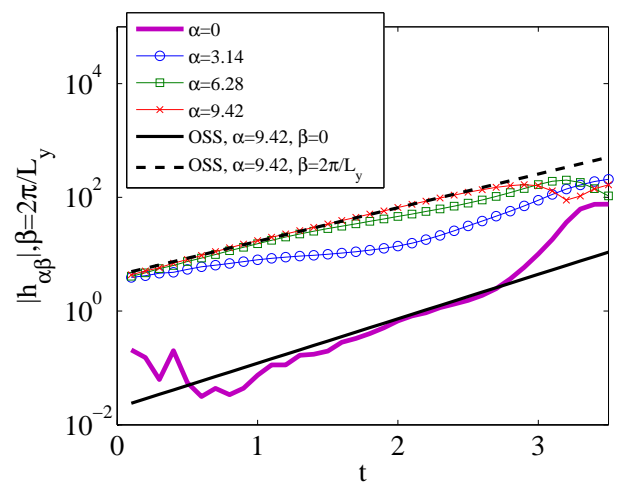

(a) P1 revisited, spanwise

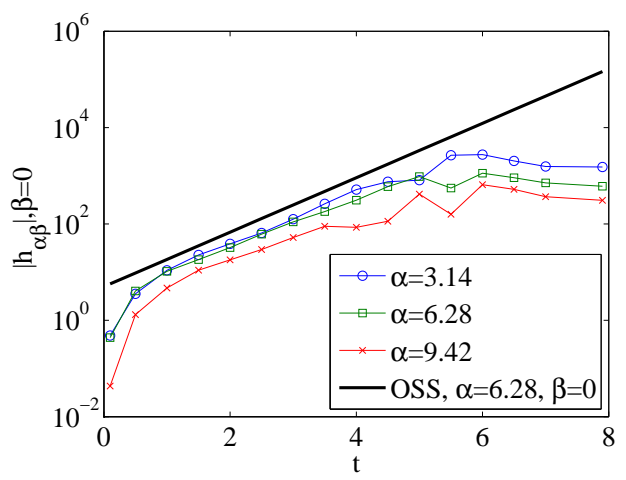

(b) P3, streamwise

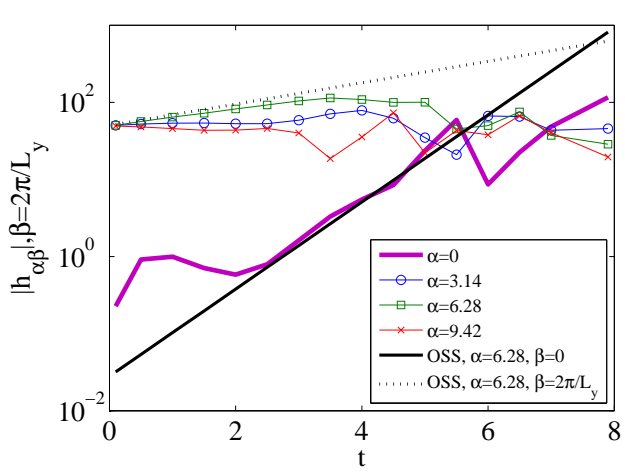

(c) P3, spanwise

Figure 17. Interfacial spectra for the cases P1, P3. (a) Case P1 revisited, streamwise modes. (b) Streamwise modes, P3 (c) Spanwise modes, P3, with $\beta=2 \pi / L_{y}$.

Equation (4.3), will be amplified at (close to) the maximal rate. By examining relevant spectra (Figure 17(a)), we have confirmed that this is indeed the case: in the simulations P1-P2 considered earlier, after some transience, the purely spanwise mode $\left(0,2 \pi / L_{y}\right)$ does indeed grow at the same rate as the most-dangerous (streamwise) mode. However, given the long transient time before such weakly nonlinear interactions enter, the simulation is utterly dominated by the modes that are linearly unstable. This reflects the strong supercriticality of this parameter set. A further simulation (P3) is intermediate between (P1,P2) and P3: both the direct mechanism and the weakly nonlinear mechanism play a role in producing the three-dimensional waves (Figure 17(b,c)).

\section{Open-flow instabilities}

We perform fully nonlinear simulations for long open channels, with $L_{x}$ between 3.16 and 8.0. The aim is to investigate the extent to which the linear theory governs the subsequent nonlinear evolution of the interfacial waves. We consider forcing that is both localized in time (impulsive forcing), or continuous in time. In the first case, the consid- 
ered initial condition is $\boldsymbol{u}=0$, and

$$
\eta(\boldsymbol{x}, t=0)=h_{0}+\left(\frac{0.02}{9}\right) \mathrm{e}^{-\left(x-L_{0}\right)^{2} /\left(2 w^{2}\right)} \sum_{i=1}^{3} \sum_{j=1}^{3} \cos \left(\frac{2 \pi x}{L_{x}} i+\frac{2 \pi y}{L_{y}} j+\varphi_{i j}\right),
$$

where $w=L_{x} / 10$ and $\varphi_{i j}$ is a random phase. On the other hand, for continuous-in-time forcing, in order to 'trip' open systems, the z-component of Equation (2.4) is modified to include a localized continuous-in-time momentum forcing term $F(\boldsymbol{x}, t)$ that is compactly supported in the $x$-direction, and contains a polychromatic mixture of $y$-modes and temporal frequencies (the Fourier modes in the mixture have equal amplitude and a random phase); specifically, we have

$$
F(\boldsymbol{x}, t)=\delta_{\epsilon}(\phi(\boldsymbol{x}, t)) \delta_{L_{x}}(x)\left[\frac{A_{0}}{N_{y} N_{T}} \sum_{i=0}^{N_{y}-1} \sum_{j=1}^{N_{T}} \cos \left(\frac{2 \pi i y}{L_{y}}+\frac{\Omega_{C j} t}{N_{T}}+\varphi_{i j}\right)\right],
$$

where

$$
\delta_{L_{x}}(x)= \begin{cases}1+\cos \left[\frac{16 \pi}{L_{x}}\left(x-\frac{1}{8} L_{x}\right)\right], & \frac{1}{16} L_{x} \leqslant x \leqslant \frac{3}{16} L_{x} \\ 0, & \text { otherwise. }\end{cases}
$$

The prefactors in Equation (5.2a) have the effect of localizing the momentum disturbance at the interface, and at $x=L_{x} / 8$. (Note, the divergence of the velocity field is not disturbed by this forcing.) Direct forcing of the interface itself at the inlet was also examined. However, the existence of spanwise waves at the inlet is inconsistent with the inlet condition $\boldsymbol{u}(x=0)=\left(U_{0}(z), 0,0\right)$. In practice, this combination of inconsistent inlet conditions led to an ill-posed problem, which failed to demonstrate grid-independence. For that reason, the forcing protocol in Equations (5.2) was preferred.

The cutoff forcing frequency in Equation (5.2a) is taken to be $\Omega_{C}=20$. For most of the parameter cases considered, this is much larger than the frequency of the linearly mostdangerous mode. However, even for those parameter cases where this condition is not satisfied, for sufficiently large amplitudes $A_{0}$, the non-passive nature (i.e. $\phi$-dependence) of the forcing in Equation (5.2a) is important, and further frequencies are generated nonlinearly, such that the 'effective forcing' contains frequencies greater than $\Omega_{C}$, including that of the linearly most-dangerous mode. Beyond the compact source region, these source-based nonlinearities play no role, except that they provide a broad spectrum of frequencies that are subsequently excited in the wave dynamics. The nonlinearities that do eventually matter for the interfacial waves are provided by the 'natural' evolution of the waves first of all in spatio-temporal linear theory and then in nonlinear theories.

Finally, we emphasize that similar results are obtained regardless of the amplitude of the forcing $A_{0}$. This is due to the instabilities that are intrinsic to the considered parameter regimes. Thus, disturbances of any amplitude will give rise to waves which are amplified strongly so that that large-amplitude waves will always be obtained. Well downstream, the wave amplitudes will be fixed by the intrinsic instability mechanisms (both linear and nonlinear), independent of the details of the forcing. Thus, the typical height perturbation will not be a definite function of $A_{0}$. This description has also been confirmed both for the simulations described herein and for further simulations that were performed to validate more carefully this description (not shown). In all cases, the asymptotic shape of the downstream three-dimensonal wave structures is more-or-less independent of the magnitude of the forcing amplitude. 


\begin{tabular}{|c|c|}
\hline Case & Parameters \\
\hline $\begin{array}{l}\mathrm{CH} 1 \\
\mathrm{CH} 2 \\
\mathrm{CH} 3 \\
\mathrm{CH} 4\end{array}$ & $\begin{array}{l}\mathcal{S}=0.001, R e=100 \\
\mathcal{S}=0.01, R e=100 \\
\mathcal{S}=0.1, R e=100 \\
\mathcal{S}=0.01, R e=200\end{array}$ \\
\hline
\end{tabular}

TABLE 3. Summary of parametric study of open flows with impulsive forcing, all with $m=30$. Geometric parameters: $\left(L_{x}, L_{y}, L_{z}\right)=(3.54,1,1)$. Forcing location: $L_{0}=1$. Each parameter study corresponds to linear absolute instability.

\subsection{Impulsive forcing}

For the impulsive initial condition, simulations were performed in those parameter regimes that are linearly absolutely unstable according to two-dimensional linear Orr-Sommerfeld theory. The qualitative picture is that the interface evolves rapidly in the downstream direction, and acquires a complicated three-dimensional structure. Again in this qualitative picture, the downstream interfacial configuration resembles very closely the periodic simulations already discussed. On the other hand, close to the source of the initial impulse, the disturbance (both in the interfacial height and the flow velocity) rapidly evolves towards a two-dimensional configuration and then first grows according to linear theory, before saturating to some finite value. At saturation, the interface evolution at the source appears to be periodic in time, with a single characteristic frequency. Finally, at very late times, the interface shape - both downstream and upstream - takes on a decidedly twodimensional character. Snapshots of the interface configuration for the particular case CH2 in Figure 18 confirm this description. A time series of the averaged wall-normal velocity $\int_{0}^{1} \mathrm{~d} z \int_{0}^{1} \mathrm{~d} y w(x, y, z, t)$ for the same case, at $x=L_{0}=1$ is shown in Figure 19 (i.e. corresponding to the initial impulse location); this further confirms the saturation and the frequency-selection discussed herein.

The behaviour exemplified in Figures 18-19 suggests that a global mode (Couairon \& Chomaz 1999) may be in operation in the system $\dagger$. The picture is that the polychromatic impulsive disturbance 'selects' the global mode at the disturbance source, leading to a two-dimensional wave evolution near the source and in the upstream direction. In the downstream direction, the polychromatic impulsive source initially leads to a complicated interfacial evolution, wherein the complicated three-dimensional ligament dynamics give rise to multiple timescales, as opposed to the single timescale inherent in the global mode. However, this is a transient effect: because the disturbance at the source evolves into a two-dimensional global mode, the same two-dimensional structure feeds the downstream disturbance at later times, meaning that the eventual interfacial dynamics are almost entirely two-dimensional (Figure 18(e)) throughout the entire domain. The same picture is replicated in the other simulations $(\mathrm{CH} 1-\mathrm{CH} 4)$.

For these reasons, we examined the frequency of the disturbance oscillation at $x=L_{0}=$

$\dagger$ For the avoidance of doubt, there is no 'global' instability in the sense of a spatially-evolving base state in the streamwise direction (we have carefully checked that the average base-state profile is translation-invariant in the $x$-direction). The 'global' instability referred to is in the sense of Couiron and Chomaz, namely a self-sustained nonlinear oscillation that bifurcates from the linearly absolutely unstable case. 


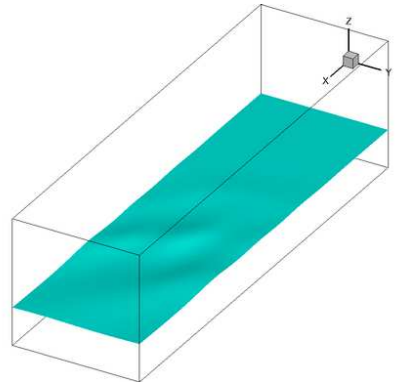

(a) $t=2.0$

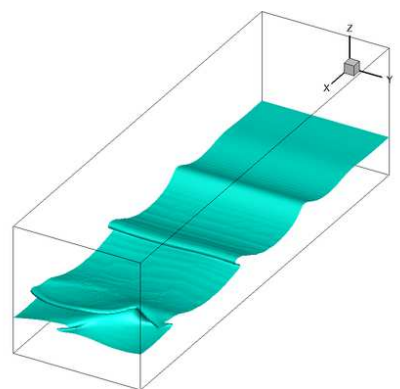

(d) $t=4.5$

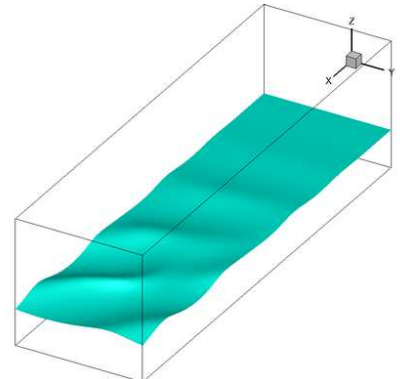

(b) $t=3.0$

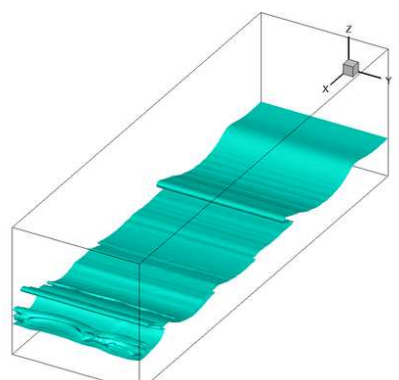

(e) $t=8.0$

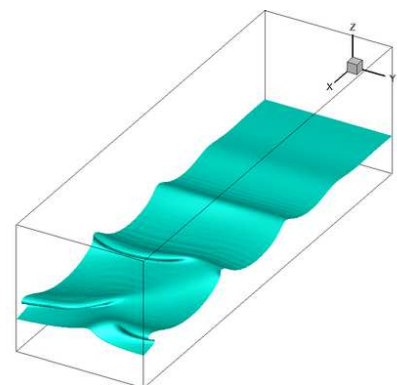

(c) $t=4.0$

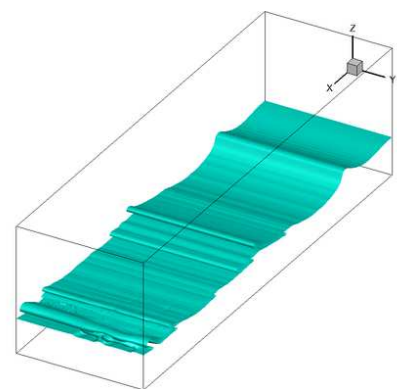

(f) $t=10.0$

FIGURE 18. Parameter study CH2: impulsively-forced case with impulsive forcing located initially at $x=L_{0}=1$.

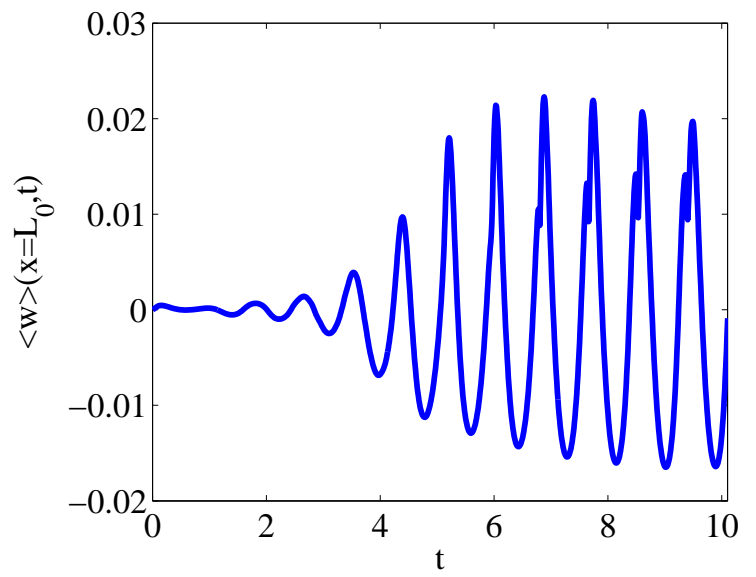

Figure 19. Averaged velocity $\langle w\rangle:=\int_{0}^{1} \mathrm{~d} x \int_{0}^{1} \mathrm{~d} y w(x, y, z, t)$, as a function of $t$ at $x=L_{0}=1$. The pertinent simulation is $\mathrm{CH} 2$.

1 as a function of the control parameter. In the present context, the control parameter was selected to be $\mathcal{S}$ : for fixed $R e=100, m=30, h_{0}=0.3$, the flow is linearly absolutely unstable for $\mathcal{S}<\mathcal{S}_{\mathrm{c}} \approx 0.69$ and linearly convectively unstable above the same threshold value. Because of the extremely long times required for saturation to occur (24 hours' runtime with 1024 cores on the machine described in Section 2), only a handful of threedimensional simulations was performed. However, this is sufficient (i) to demonstrate that the upstream dynamics of the three-dimensional system are governed by the twodimensional global mode, and that (ii) the downstream dynamics as $t \rightarrow \infty$ are governed 


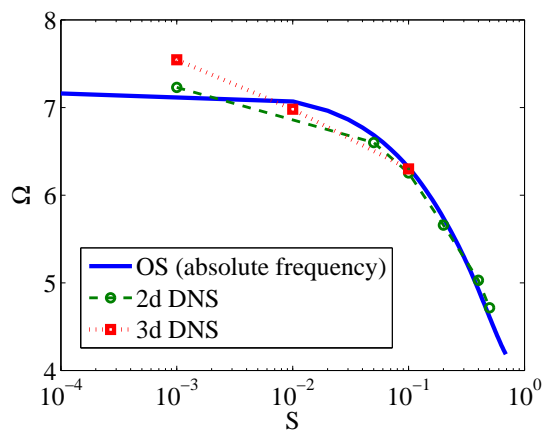

(a)

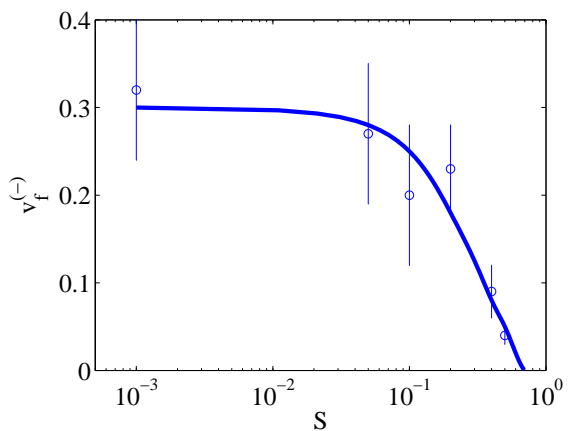

(b)

FIGURE 20. (a) Measured global-mode frequency from DNS (both 2D and 3D), compared to the linear absolute (2D) frequency. The frequency is extracted from the time series of the averaged velocity $\int_{0}^{1} \mathrm{~d} x \int_{0}^{1} \mathrm{~d} y w(x, y, z, t)$ at location $x=L_{0}=1$. (b) Velocity of upstream-propagating front: comparison between linear theory and 2D DNS. Note: the upstream-propagating front is eventually frozen-in, due to the Dirichlet boundary condition at the inlet.

by the same; characterization of the global mode can subsequently be performed using less intense two-dimensional simulations. The result of the foregoing quantitative approach is summarized in Figure 20. In Figure 20(a) it is seen that the upstream dynamics of the full three-dimensional simulations are governed by a two-dimensional nonlinear global mode, while the selected frequency of the two-dimensional global mode is very close to the linearly absolutely unstable frequency (the small difference between the $2 \mathrm{D}$ and $3 \mathrm{D}$ cases is due to the shorter time interval over which the 3D data are gathered; this difference was observed to decrease upon increasing the 3D simulation time).

The close match between the linearly absolutely unstable frequency and that of the global mode suggests that the upstream-propagating front is a 'pulled front', in other words, that the global-mode properties are determined entirely by the linear dynamics (Huerre 2000; Chomaz 2003). To confirm this hypothesis, we computed the velocity of the upstream-propagating front. For reference: the upstream- and downstreampropagating fronts represent the maximum extent of the spatially-localized disturbance at a given time. The location of the upstream-propagating front becomes frozen after a finite time because of the influence of the Dirichlet boundary condition at $x=0$. In any case, the front velocity before this final time is obtained from the full (two-dimensional nonlinear DNS) and the result is compared with the front velocity from linear theory. The front velocity from linear theory is computed using the method of Ó Náraigh et al. (2013). Concerning the nonlinear DNS, it was not possible to locate the front at a given time exactly in the DNS; however, by considering the spacetime plot of $\int_{0}^{1}|w(x, z, t)|^{2} \mathrm{~d} z$, a range of possibilities for the front location (hence front velocity) was obtained. This is reflected in the error bars in Figure 20. In any case, the location of the upstreampropagating front in the nonlinear DNS agrees with the same front velocity in linear theory, confirming the hypothesis that the global mode arises from a 'pulled front'. Further consideration of the so-called 'healing length' (Couairon \& Chomaz 1999) did not provide any further means of characterizing the global mode. This is not surprising, as simple scaling laws for the healing length as a function of the criticality parameter $\epsilon$ (in this case, $\left.\epsilon=\left|\mathcal{S}-\mathcal{S}_{\mathrm{c}}\right|\right)$ are known to break down when the dispersion relation of the linear theory is different from the simple case that pertains for the complex Ginzburg-Landau equation (Selvam et al. 2009). 


\begin{tabular}{l|ll|l|}
\hline Case & Physical parameters & Geometric parameters & $\mid$ Figure \\
\hline \hline CH5 & $A_{0}=100, \mathcal{S}=0.01, R e=100$ & $\left(L_{x}, L_{y}, L_{z}\right)=(3.16,1,1)$ & Figure 21 \\
CH6 & $A_{0}=300, \mathcal{S}=0.1, R e=100$ & $\left(L_{x}, L_{y}, L_{z}\right)=(8.0,1,1)$. & Figures 22-23 \\
CH7 & $A_{0}=300, \mathcal{S}=0.1, R e=300$ & $\left(L_{x}, L_{y}, L_{z}\right)=(8.16,1,1)$. & \\
\hline
\end{tabular}

TABLE 4. Parametric study showing the effects of varying the surface tension, all with $m=30$.

Finally, we have verified that increasing the amplitude beyond that in Equation (5.1) and running simulations in a linearly convective regime does not trigger self-sustained oscillations. This confirms once again the fact that the self-sustained oscillations (both linear and nonlinear) are triggered by the linear absolute instability. We have also verified tentatively (e.g. CH4) that these results carry over to different parameter regimes. However, a more complete parametric study is performed in the context of continuousin-time forcing, to which the global-mode theory just described also applies, as we now demonstrate.

\subsection{Continuous forcing}

We perform fully nonlinear simulations for long open channels, with $L_{x}$ between 3.16 and 8.0. The aim is to investigate whether the influence of the global mode carries over from the impulsive forcing to the continuous-in-time case. The relevant simulations are summarized in Table 4 and Figures 21-23.

In Figures 21-23, the ligament formation occurs with a remarkable regularity. To investigate this further, we track back the origin of the ligaments via the total interfacial curvature $\kappa_{x}(x, t)$, defined here as

$$
\kappa_{x}(x, t)=\max _{y, z}(\nabla \cdot \hat{\boldsymbol{n}}) .
$$

The results are plotted in the $(x, t)$ plane in Figure 26. We have also examined a further curvature-related quantity, namely $\max _{y, z} \nabla_{2} \cdot\left(\nabla_{2} \phi /\left|\nabla_{2} \phi\right|\right)$, where $\nabla_{2}=\left(\partial_{y}, \partial_{z}\right)$ denotes the gradient operator restricted to the $(y, z)$-plane. A spacetime plot based on this further curvature-related quantity yields a nearly-identical picture (not shown). For all three plots in Figure 26, the narrow strips in spacetime where the curvature initially develops a large magnitude can be related to the formation of interfacial waves, as is readily verified by comparison with the interfacial snapshots, either in Figure 21 for $\mathrm{CH} 5$, or Figures 2223 for CH6. These narrow strips broaden into much wider regions of spacetime where the curvature attains an even larger magnitude. These regions are similarly associated with ligaments.

The spacetime diagrams in Figure 26 demonstrate conclusively the regularity with which wave/ligament structures occur in all three parameter cases, suggesting that a frequency-selection criterion is at work. To investigate the frequency selection, we first of all examine parameter cases corresponding to linear absolute instability $(\mathrm{CH} 5, \mathrm{CH} 6)$. The frequency of ligament formation was found by examining a time series $\kappa_{x}(x, t)$, at a fixed location $x$. The frequencies were extracted by eye from Figure 26 and the results were subsequently verified by a full spectral analysis of $\kappa_{x}(x, t)$ by carrying out Fourier transforms with respect to $t$ at fixed $x$-locations. The frequencies are reported in Table 5 


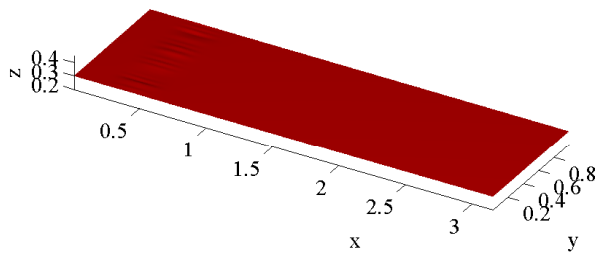

(a) $t=0.1$

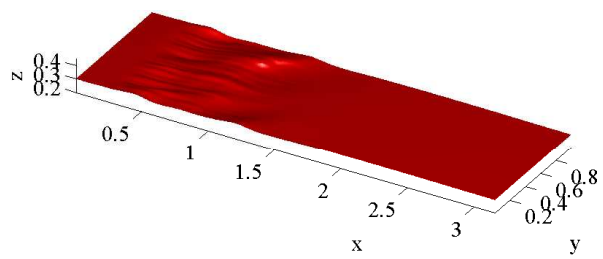

(c) $t=1.6$

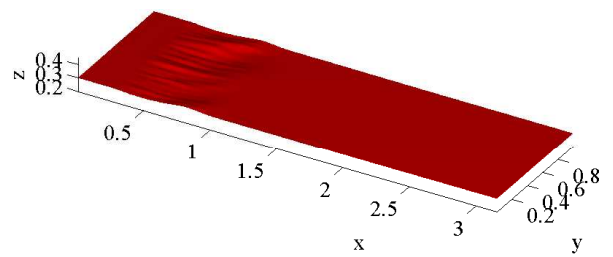

(b) $t=0.9$

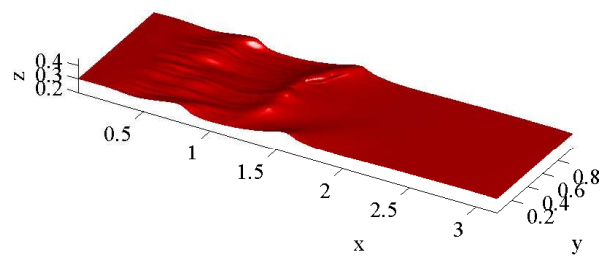

(d) $t=2.2$

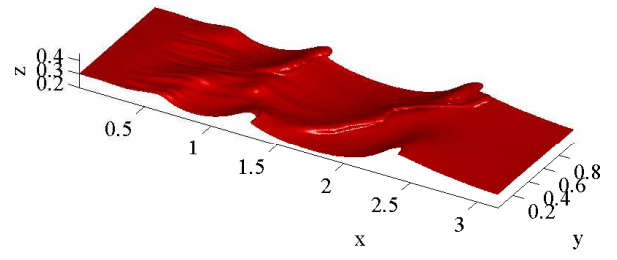

(e) $t=2.9$

FIgURE 21. DNS for high-surface-tension case CH5 - snapshots of interface at various times.

and are those obtained by the spectral analysis and the error bound in the measured frequency is half the sampling frequency.

The results in Table 5 for $\mathrm{CH} 5-\mathrm{CH} 6$ are explained in combination with Figures 21-23. We focus on intermediate $x$-stations where the ligaments are on the verge of forming, yet sufficiently far from the forcing so that the flow responds to the forcing via intrinsic mechanisms, i.e. so that the precise details of the momentum forcing term are not important. In this region, and at late times, the waves are of finite amplitude by definition, wherein linear theory would not necessarily apply. However, the measured selected frequency 


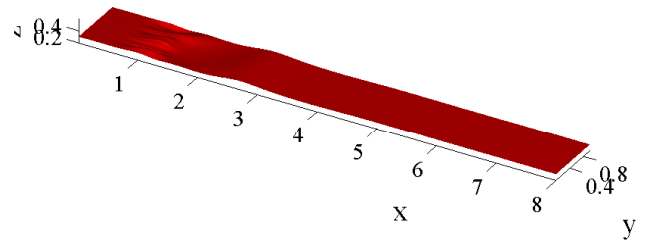

(a) $t=1.5$

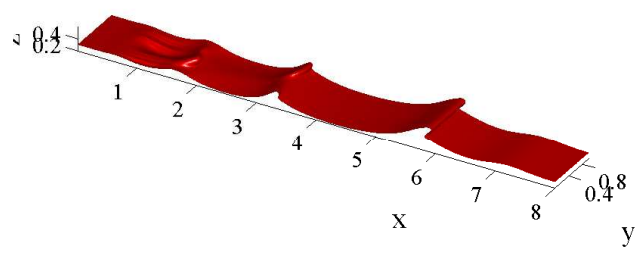

(c) $t=3$

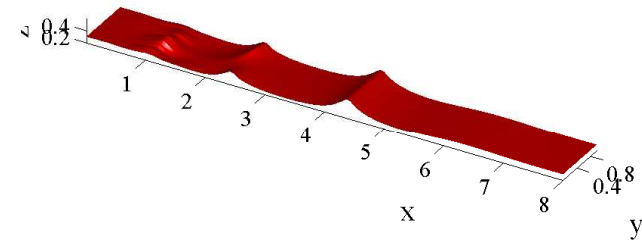

(b) $t=2.5$

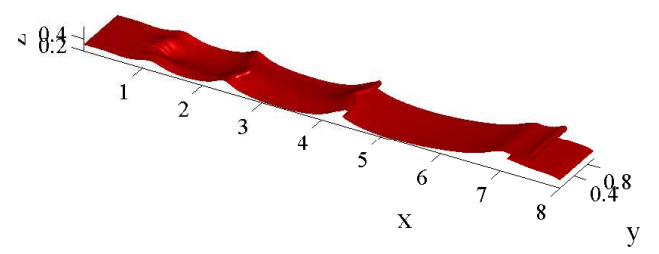

(d) $t=3.5$

FiguRE 22. DNS for high-surface-tension case CH6 - interface height at various early times.

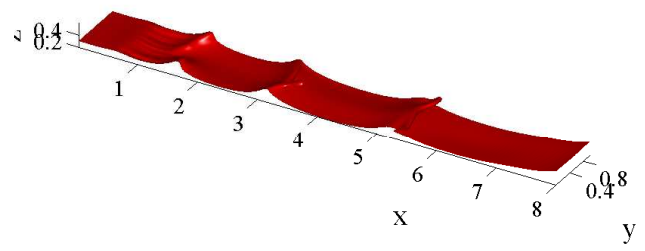

(a) $t=4$

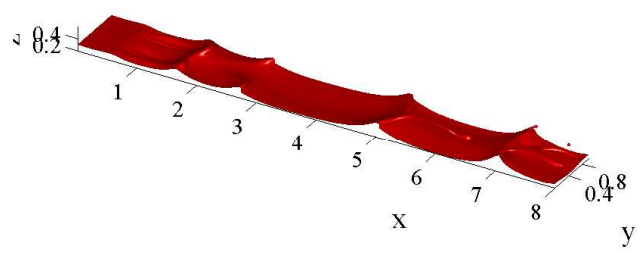

(c) $t=5$

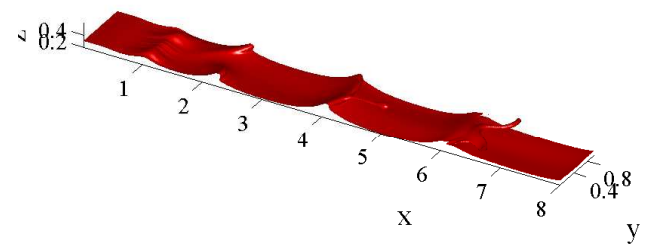

(b) $t=4.5$

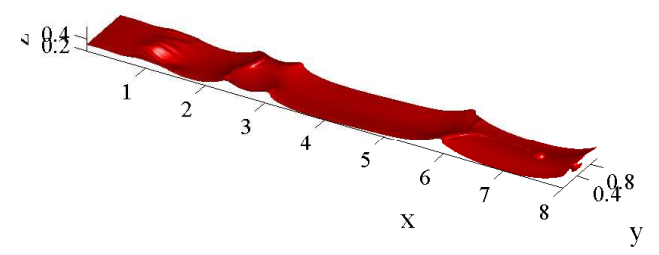

(d) $t=5.5$

FiguRE 23. DNS for high-surface-tension case CH6 - interface height at various later times.

at the relevant $x$-stations does in fact agree rather closely with the linearly absolutely unstable frequency, which in turn agrees closely with the two-dimensional global-mode frequency. Concerning the frequency selection via linear absolute instability, this is standard (e.g. the reference by Huerre (2000)), while the persistence of the same frequency at larger amplitudes confirms the presence of a global mode whose properties are entirely governed by linear theory (such global modes arise from 'pulled' fronts, as previously demonstrated in the simulations with impulsive forcing). The selected frequency is almost exactly the same in both the linear and nonlinear cases: thus, a single frequency (or at most, a very narrow band of frequencies) is 'locked in' to the system at the rel- 


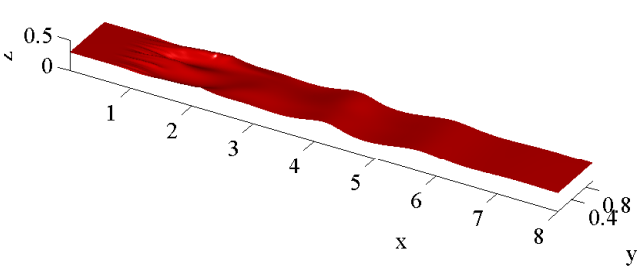

(a) $t=0.525$

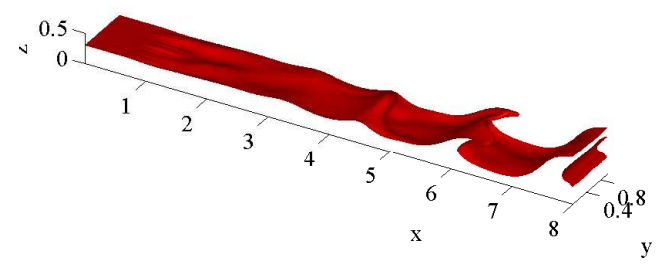

(c) $t=0.75$

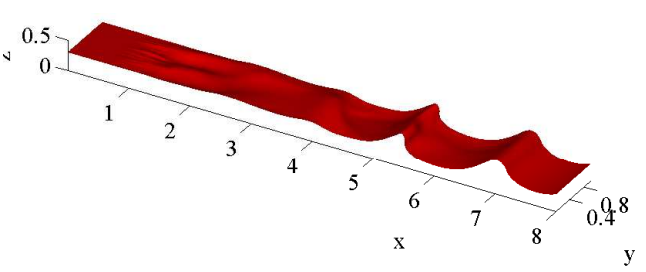

(b) $t=0.675$

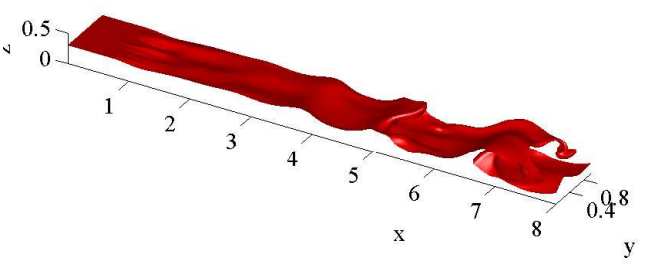

(d) $t=0.825$

FiguRE 24. DNS for the CH7 case $(R e=300, \mathcal{S}=0.1)$ - interface height at various early times.

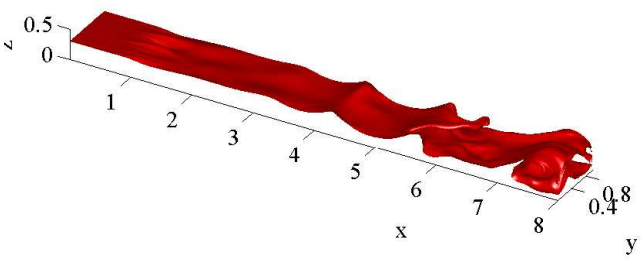

(a) $t=0.9$

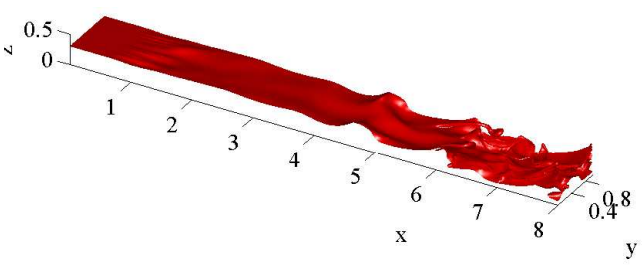

(c) $t=1.125$

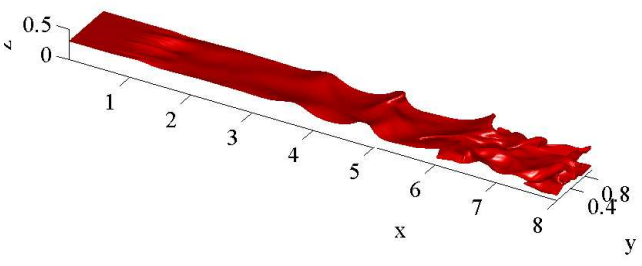

(b) $t=0.975$

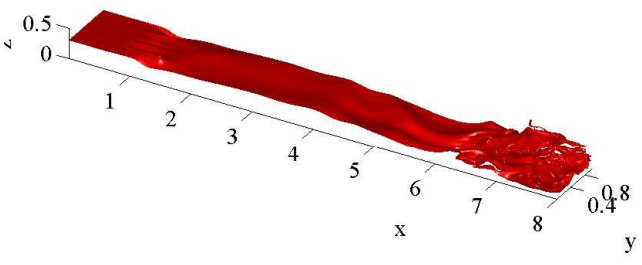

(d) $t=1.35$

Figure 25. DNS for the CH7 case $(R e=300, \mathcal{S}=0.1)$ - interface height at later times.

evant $x$-stations, and disturbances at the same frequency are then carried downstream, where secondary instability precipitates the formation of three-dimensional structures. Because of the externally-imposed forcing, the disturbance source retains spanwise waves indefinitely (this is in contrast to the periodic case with impulsive forcing, where the disturbance source eventually took on a two-dimensional structure, due to the selected two-dimensional global mode there). Thus, for the externally-forced open flow, spanwise perturbations are generated continuously, and are constantly carried downstream, 


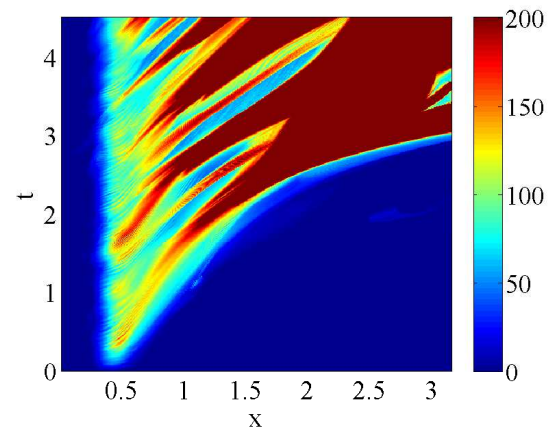

(a)

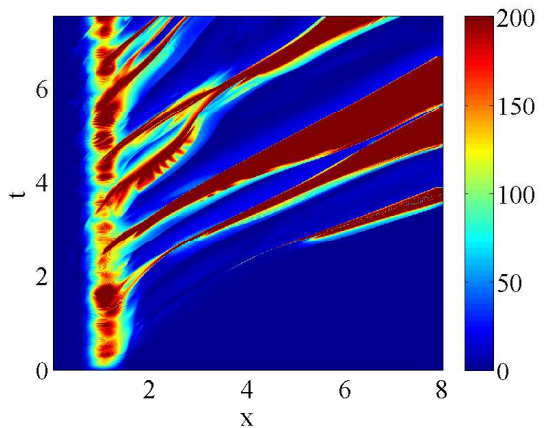

(b)

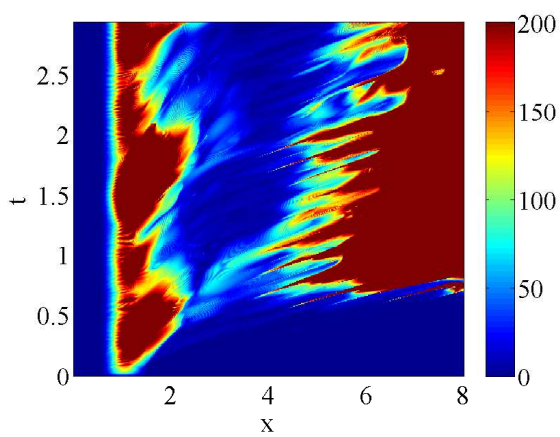

(c)

Figure 26. The maximum curvature in $(y, z)$ planes versus $(x, t)$ for Cases CH5 (a), CH6 (b), and $\mathrm{CH} 7(\mathrm{c})$.

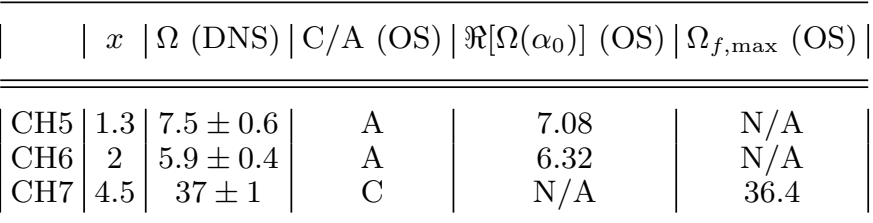

TABLE 5. Frequency of ligament generation for three distinct parameter cases, together with comparisons against spatio-temporal (two-dimensional) OS theory. For cases CH5-CH6, the spatial growth rate $-\alpha_{\mathrm{i}}\left(\alpha_{\mathrm{r}}\right)$ admits no maximum away from $\alpha_{\mathrm{r}}=0$, meaning that it is impossible to compute a maximum frequency $\Omega_{f, \max }$.

such that secondary disturbances (and hence ligament formation) are underpinned by the more dominant two-dimensional waves. In this instance, the two-dimensional waves possess a frequency determined by the rules outlined above and hence, the frequency of ligament formation is inherited from the supporting two-dimensional waves.

Consideration is also given to the convectively unstable parameter set (CH7). Again, as demonstrated by Figure 26 and Table 5, there is a definite frequency of ligament formation. Naturally, this cannot be explained by the absolute instability / global-mode theory used in the context of the simulations $\mathrm{CH} 5-\mathrm{CH}$. Instead, we revert to theory of linear 


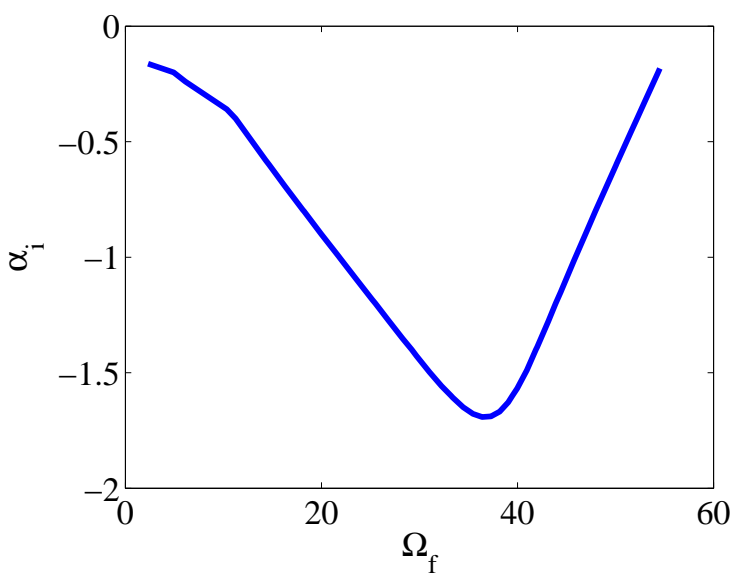

FIGURE 27. Downstream spatial growth rate as a function of forcing frequencies (OS analysis). Here $(R e, m, \mathcal{S})=(300,30,0.1)$, corresponding to a convectively unstable case.

spatial growth. Again also, it suffices to consider the two-dimensional scenario, since the most-prominent waves are two-dimensional (of coure, well downstream, three-dimensional behaviour is observed). In this instance, the generic asymptotic streamfunction response to localized continuous-in-time forcing $\delta(x) \mathrm{e}^{-\mathrm{i} \Omega_{f} t}$ is given by (e.g. Huerre (2000))

$$
\psi(x, t) \sim \mathrm{i} H(x) \frac{\mathrm{e}^{\mathrm{i}\left[\alpha^{+}\left(\Omega_{f}\right) x-\Omega_{f} t\right]}}{\left.\frac{\partial D}{\partial \Omega}\right|_{\left(\alpha^{+}\left(\Omega_{f}\right), \Omega_{f}\right)}}-\mathrm{i} H(-x) \frac{\mathrm{e}^{\mathrm{i}\left[\alpha^{-}\left(\Omega_{f}\right) x-\Omega_{f} t\right]}}{\left.\frac{\partial D}{\partial \Omega}\right|_{\left(\alpha^{-}\left(\Omega_{f}\right), \Omega_{f}\right)}}, \quad t \rightarrow \infty,
$$

where $D(\Omega, \alpha)$ denotes the dispersion relation obtained from the unforced normal-mode eigenvalue problem for the streamfunction $\psi(x, t)$, and where $\alpha^{ \pm}\left(\Omega_{f}\right)$ denotes the spatial growth rates associated with downstream propagation (plus sign) and upstream propagation (minus sign), derived from the same dispersion relation. One may extend this result if a combination of forcing frequencies $\left\{\Omega_{f 1}, \cdots, \Omega_{f n}\right\}$ is present. Using the linearity of the small-amplitude streamfunction equation, the response in Equation (5.4) will consist of a sum over all forcing frequencies. In the convectively unstable case, the dominant frequency well downstream of the forcing will be that frequency $\Omega_{f, \max }$ that maximizes the pertinent spatial growth rate, i.e. $\Omega_{f, \max }$ corresponds to the most negative spatial wave number in the set $\left\{\alpha_{i}^{ \pm}\left(\Omega_{f 1}\right), \cdots, \alpha_{i}^{ \pm}\left(\Omega_{f n}\right)\right\}$ (Figure 27 ). This prediction holds up in the simulation: the measured frequency of ligament formation is $\Omega=37 \pm 1$, while the forcing frequency that most enhances downstream spatial growth according to OS theory is $\Omega_{f, \max }=36.4$. The conclusion therefore is that linear theory selects a frequency from the range of forcing frequencies available from the source. Disturbances are convected downstream at the linearly selected frequency, whereupon three-dimensional structures feed off the linearly-selected two-dimensional wave, leading to ligament formation at the two-dimensonal wave frequency.

The selectivity observed in the convectively unstable case was not observed in the work of Valluri et al. (2010). The mechanism just described in Equation (5.4) and in the subsequent text has however been discussed previously by Selvam et al. (2009), and some evidence of the same phenomenon has been found therein. A possible explanation for the very distinct frequency-selection for the ligament formation in the present convectively unstable case is that the dispersion relation $\alpha_{\mathrm{i}}^{+}\left(\Omega_{f}\right)$ possesses a sharp maximum at $\alpha=36.4$ (e.g. Figure 27), meaning that the system is able to discriminate between that 
narrow band of frequencies that promotes spatial growth in the downstream direction, and all other forcing frequencies.

\section{Ligament formation and dynamics/kinematics in open flows}

Having investigated the onset of ligament formation in the previous section, we study the late-time dynamics of ligaments here. We have performed a parametric study using nonlinear DNS for $50 \leqslant R e \leqslant 300,0.01 \leqslant \mathcal{S} \leqslant 0.3$, mostly at $m=30$, which includes a $\mathrm{C} / \mathrm{A}$ transition of the corresponding $2 \mathrm{D}$ system at sufficiently large $\mathcal{S}$ or $R e$. We have found that sharp flow-regime boundaries (between elongated ligaments and sheets) cannot be drawn: simulation results indicate that sheet-like disturbances can eventually exhibit thread-like ligaments, and both types of behaviour can co-exist. Furthermore, the observed late-time dynamics of ligaments is affected by additional parameters: the strength of the forcing, the length of the computational domain and the duration of the simulations. Overall though, two general trends can be reported. First, ligaments tend to be more sheet-like at low values of $\mathcal{S}$ (although thread-like features can occur further downstream), suggesting a passive stretching of overturned waves without significant effects of surface tension. A second general trend is that wave overturning and ligament formation develop less frequently and at a slower pace at larger values of $\mathcal{S}$. In this section, we characterize the growth of ligaments and report flow behaviour involving many ligaments.

\subsection{Ligament growth}

We first study the distance between tip and foot of ligaments in Figure 28(a). The timescale of growth is more or less the same in all cases (including varying the surfacetension parameter $\mathcal{S}$ and the viscosity ratio $m$ ), with the notable exception of cases wherein the Reynolds number, $R e$, is varied. The fact that an increase in the value of $\mathcal{S}$ by an order of magnitude hardly affects the ligament length as a function of time is incompatible with the governing physical mechanism being the work done by tangential shear stress being converted into surface energy, as in a droplet stretched whilst pinned on a wall in shear flow (e.g. Ding et al. (2010)). Furthermore, the ligament dynamics argument of Marmottant \& Villermaux (2004) for gas-assisted jets, when modified such that a rate of change of momentum of a ligament corresponds to the shear stress integrated over the ligament (rather than normal stress), would lead one to expect a significant dependency in Figure 28(a) on the value of $m$, which is not observed.

The results in Figure 28(a) suggest instead that these ligaments are elongated in a kinematic way, in line with the 'strong-flow' regime of droplet stretching in the work by Cristini et al. (2003). A vector $\mathbf{L}$ is advected passively approximately according to Batchelor (1967) (Chapter 3),

$$
\frac{d L_{i}}{d t}=L_{j} \frac{\partial u_{i}}{\partial x_{j}} .
$$

where it is assumed that the vector length is small compared to length scales over which $\nabla \mathbf{u}$ varies. We first observe that a unidirectional flow (over a flat interface) is independent of $\mathcal{S}$, and that the corresponding dimensionless shear rate just above the interface is hardly affected by the value of $m$ but varies approximately linearly with the value of $R e$. The large discrepancy in time scale seen in Figure 28(a) for $R e=300$ (with $\mathcal{S}=0.1$ ) compared with the cases at $R e=100$ can largely be explained by kinematic elongation of ligaments: after multiplying the time variable by $R e$, the velocity of elongation for $R e=$ 300 differs only by a factor of about two from the other data. This is strengthened further 


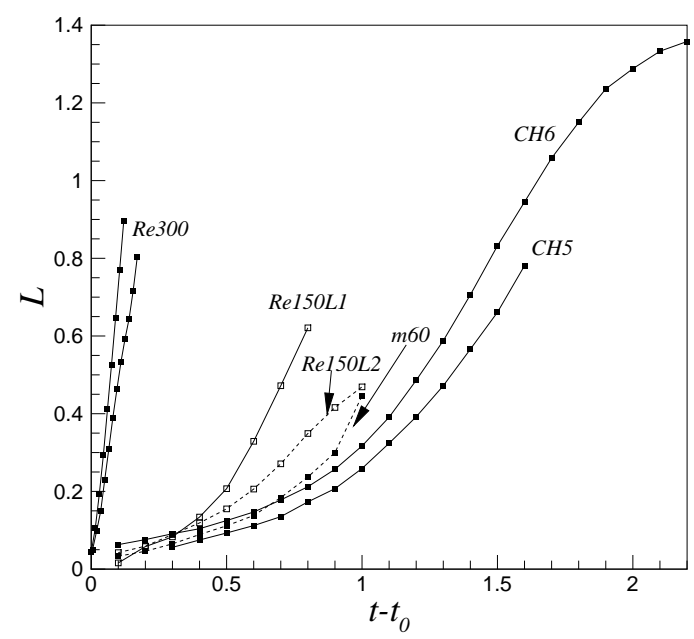

(a)

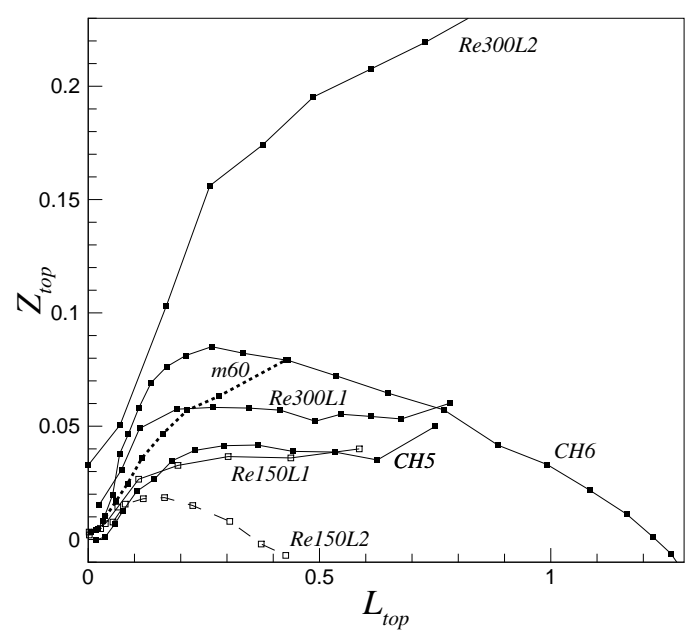

(b)

Figure 28. (a) Ligament length versus time; (b) Position of the top of the ligament tip relative to the interface above the foot. Various cases are considered, as indicated. In CH5 and $m=60$, the ligaments are mostly thin sheets, whereas that traced in CH6 is a highly elongated thread. For $R e=150$, two ligaments have been traced, both being thread-like, that labelled Re $150 L 1$ being rather isolated, that labelled Re $150 L 2$ being surrounded by other ligaments. For $R e=150$, two ligaments have been traced: one being thread-like (labelled Re300L1), the other sheet-like (labelled Re300L2). The data are shown up to the point of tearing or breakup.

by additional simulations included in Figure 28(a) for ligaments at $R e=150, \mathcal{S}=0.01$ (a snapshot of this simulation is shown in Figure 1). On the other hand, there is scatter in the growth rates of ligaments in the same simulation, to which we return below.

In fact, Equation (6.1) explains further details of the behaviour of ligaments in these flows. Two regimes are seen in Figure 28(a): an early-time behaviour, which further 
inspection of our data shows to be near-exponential in time, and a near-linear regime. Although such behaviour was also observed in the two-dimensional simulations in the work by Valluri et al. (2010), no further analysis was offered there. In Figure 28(b), it is seen that at early times (up to the end of a near-exponential time dependency in Figure 28(a)), the relative position of the interface above the tip moves upwards relative to the interface above the ligament foot (we have found this to be caused mostly by a downwards motion of the latter, which would be expected from the foot - initially a large-amplitude wave - is drained to form part of the ligament). The largest variation in the velocity field is normal to the interface, and from Equation (6.1), this component of $\mathbf{L}$ is expected to increase exponentially. Subsequently, this component of $\mathbf{L}$ saturates, rendering the right-hand side of Equation (6.1) constant, thereby resulting in linear elongation. Therefore, ligaments that are not stretched significantly in the $(z-)$ direction of the main variation of the flow are expected to be extended less in the main flow direction. This mechanism is not unlike Taylor dispersion for passive scalars, wherein a small rate of diffusion of a passive scalar in the direction of the main variation of the velocity field leads to large dispersion in the main flow direction (Taylor 1953). In conclusion, the results shown in Figure 28(a) and (b) are best understood together: cases wherein the extension in the $z$-direction is small lead to weaker extension in the $x$ - (main flow) direction. The variation in the growth rates among ligaments in the same simulation in Figure 28(a) is to be expected given their differences in Figure 28(b): for example, the ligament labelled Re150L1 grows rather quickly in Figure 28(a) when compared to Re150L2, but in the latter is seen in Figure 28(b) to descend into a trough whereas the former maintains its 'posture'.

Finally, the detailed parameter study carried out in the present section enables one to connect the results somewhat to the energy-budget anlysis in the framework of linear theory. The parameter cases that admit absolute instability correspond to more interfacial-type modes, driven by the Yih instability, while the parameter cases that are convectively unstable correspond to more shear-type modes (albeit that a small contribution to the perturbation energy from the interfacial viscosity mismatch is always present). The parameter cases considered show that increasing the Reynolds number promotes the shear-type mode while reducing the interfacial or Yih-type mode. Since the shear-type modes are typically unconditionally convectively unstable, the same parameter governs the transition between absolute and convective instability. Of course, the parameter space in the present problem is rich, and we have already demonstrated that the inverse capillary number also controls the absoluteness or otherwise of the instability. In any event, the nonlinear DNS results of this section confirm that the frequency selection criteria and ligament generation are independent of the precise details of the pertinent underlying linear mode and depend only on the more coarse-grained features, such as absolute and convective instability, and the kinematic description of the ligament generation and evolution.

\subsection{Detailed study of the case $\mathrm{CH}^{7}$ and discussion of interfacial 'turbulence'}

Finally, we consider in more detail some simulation results concerning the simulation CH7 $(R e=300, \mathcal{S}=0.1)$. Referring to Figures $24-25$, the early-time $t \lesssim 0.675$ results are similar to those observed before for $(\mathrm{CH} 5, \mathrm{CH} 6)$ : the disturbances downstream of the forcing region are largely two-dimensional, with three-dimensional perturbations superimposed on the crest of large-amplitude two-dimensional waves. These waves are stretched into sheet-like ligaments reminiscent of those seen in the case CH5 (e.g. the large three-dimensional overturned wave at $x \approx 6$, at $t=0.75$ ). As the ligament is stretched by the mean flow, it 'rolls up' and is carried out of the domain. However, these events are 


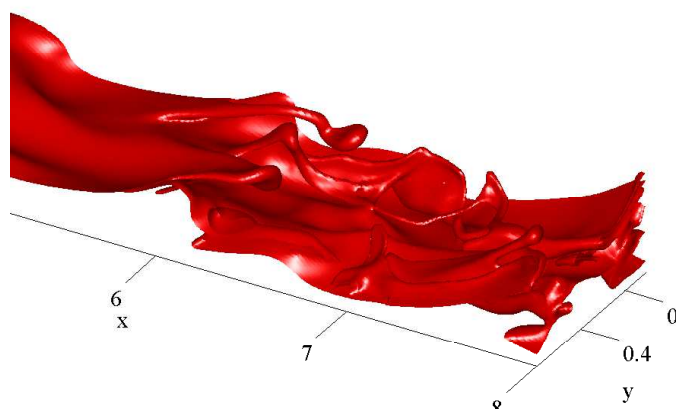

Figure 29. Snapshot of the interface height at $t=1.125$, enlarged with respect to the previous figure to show the co-existence of 'sheets' and ligaments.

accompanied by a violent collision on the windward side between the ligament's carrier wave and a neighbouring ligament just upstream (e.g. $t=0.9,0.975$ ). Such collisions continue indefinitely, leading to a complicated 'turbulent' interfacial structure near the outlet.

Although irregular flow behaviour is observed in prior work (Valluri et al. 2010; Fuster et al. 2009), its relation to a weakly-perturbed state is unclear from these studies. In fact, the transition to a highly-agitated state observed in Figure 25 for a three-dimensional system appears within a very short distance downstream from the region where waves are still of small amplitude. The basic description given above is supported by a further simple kinematic argument. For CH7, the frequency of ligament generation is five times greater than that of CH5 (see Table 5). In addition, due to the convective nature of the instability in $\mathrm{CH} 7$, the ligaments form only well downstream in response to a spatial amplification of the localized forcing (see Table 5 and Figure 24-25). Thus, a large number of ligaments is created very rapidly in a small part of the domain. In addition, these ligaments are distorted by the mean flow, wherein the mean shear rate on the gas side is three times larger for $\mathrm{CH} 7$ compared to $\mathrm{CH} 5$ (the mean shear rate is directly proportional to the Reynolds number). Consequently, the extreme nonlinear structures in the flow are 'bunched up' and interact to form the extremely complicated structures seen at late times in Figure 25.

Finally, a detailed snapshot of the interfacial structures is shown in Figure 29, for $t=1.125$. This figure confirms the coexistence of 'sheets' and ligaments, which were observed previously in separate instances, in $\mathrm{CH} 5$ and $\mathrm{CH} 6$ respectively. In certain gasliquid systems (Marmottant \& Villermaux 2004), elongated liquid sheets (similar to those described herein) are inflated with gas from the upper layer to produce bag-like shapes that subsequently break up to produce droplets. This mechanism is typically contrasted with the scenario wherein finger-like ligaments that extend in the streamwise direction break up into droplets (Azzopardi 2003; Marmottant \& Villermaux 2004). However, Figure 29 demonstrates that both phenomena may coexist, since both 'sheets' and elongated ligaments are visible therein. These numerical findings are consistent with experimental results (Lecoeur et al. 2010), albeit for a density-contrasted system. 


\section{Discussion and conclusions}

The initial motivation for the present study was to use fully nonlinear, three-dimensional numerical simulations to identify which nonlinear mechanism should dominate beyond the point where linear theory loses validity, and by what mechanism droplets are eventually formed, as a necessary basis for a future theoretical nonlinear studies, given the diversity of types of nonlinear analysis that are available. The results for initially weaklyperturbed flows have been found to mostly follow linear theory nearly up to the point of wave overturning. For a case wherein spanwise modes are linearly stable, a subsequent nonlinear mechanism for growth has been identified and modelled theoretically, whereby spanwise modes are enslaved by the dominant streamwise mode(s). This has also been demonstrated to occur in a case wherein spanwise modes are linearly unstable, although there the spanwise modes also eventually interact, to result in more involved nonlinear behaviour.

A further candidate route to three-dimensional instability was mooted in the introduction, namely secondary instability. In this scenario, the linearly-most-dangerous streamwise mode would stabilize at large amplitude and thereafter, a finite-amplitude unidirectional travelling wave superimposed on the base state would become the 'new' base state. This new base state could itself prove unstable to three-dimensional perturbations. If secondary instability is understood in this narrow sense, namely as the formation of secondary waves on a nonlinear but non-overturned wavy base state, and exemplified by the kind of Floquet analysis performed by Schmid \& Henningson (2001), then such instability is ruled out in the present system, as the interfacial waves (both two- and three-dimensional) turn over and form complicated highly nonlinear structures, rendering analytic and quasi-analytic Floquet analyses around a wavy nonlinear base state impossible. However, if one understands secondary instability more broadly, as an instability that is fuelled by an underlying linearly unstable wave formation, then the phenomena observed in this work can be regarded as a secondary instability. Consequently, the weak nonlinear spanwise instability which is the cornerstone result of this work is an instability of the secondary type.

Regarding the flow behaviour of waves that have overturned, space-time plots of the interfacial curvature have revealed regular formation of ligaments, the frequency of which has been related to linear theory and global-mode theory in a manner that hinges upon whether the system is absolutely or convectively unstable. The results from a parametric study of the late-time evolution of ligaments and sheets have been demonstrated to support a purely kinematic explanation of ligament stretching, rather than a dynamic, force-balance-based one, and to be related to a regime of rapid stretching of droplets in extensional flow. In a case for an elevated value of the Reynolds number, a sudden transition to an agitated, strongly chaotic regime has also been presented. This sudden transition has been discussed in the context of the increased frequency at which ligaments are formed in that case.

Finally, we comment briefly on some outstanding issues and discuss possible extensions to the current work. The simulations performed so far have involved three-dimensional channels where the extent of the channel in the spanwise direction is $L_{y}=0.5,1$, and where the results were qualitatively similar for both of these geometries. However, one may estimate the effect of widening the channel further beyond $L_{y}=1$ : in this situation, a larger number of spanwise modes comes into play in linear theory, some of which will be more unstable than those present in the current simulations. Thus, in wider channels, the three-dimensional effects could become more prominent (depending of course on the choice of parameters). However, this scenario involves a mere strengthening of one of the 
routes to three-dimensional waves discussed in the paper (namely the direct route via spanwise linear instability), and the results in such a scenario are therefore expected to be qualitatively similar to the ones already obtained. The indirect weakly nonlinear route depends strongly on a coupling of a two-dimensional mode to purely spanwise modes, and it is expected that this route would also be modified only in a qualitative fashion by going over to wider channels.

The density-matched model simulated in this paper has been selected for its simplicity. Also, it was motivated by our initial practical modelling concern in the removal of viscous soils in plants during cleaning and product turnover operations (Valluri et al. 2010). Nevertheless, the considered parameter regimes have already revealed a wide range of behaviours, including mode competition and a transition between absolute and convective instability. Concerning other available variable parameters, the mean location of the interface is an important parameter, and variation of this parameter does indeed lead to mode competition (Ó Náraigh et al. 2011), and also controls the transition between convective and absolute instability (Valluri et al. 2010). The mean interface location also determines determines precisely which sub-type of the viscosity-contrast mechanism is at work (Govindarajan \& Sahu 2014; Charru \& Hinch 2000). However, in the present simulations, it is not possible to perform a complete parameter study, due to the enormous cost of the computational resources deployed. For that reason, we have focused to a certain extent on a single parameter for study, namely the surface-tension parameter (although dependence on the viscosity ratio and the Reynolds number is also addressed in some depth). This was for two reasons. First, the surface-tension parameter is a convenient 'switch' that controls both the transition between absolute and convective instability, but also the shape of the resulting ligaments. Also, focusing on the single parameter $h_{0}=0.3$ was again motivated by the original practical application in industrial cleaning processes in liquid-liquid flows. Certainly, a parameter study based additionally on $h_{0}$ will throw up some interesting features. However, the methods developed in this paper (the weak nonlinear theory whereby the transient mode is excited nonlinearly and selects a given excitation on the basis of linear transient-growth theory, as well as the kinematic analysis of the ligaments) are totally generic, and should be applicable more broadly. Thus, it is important to place these methods on a firm evidence-based footing, so they can be applied with confidence to wider parameter studies in the future. Indeed, it will be of compelling interest to extend the present analyses to systems residing in other distinct parameter regimes, thereby widening unambiguously both the applicability of the present findings and the scope of the high-performance computing model developed in this paper.

\section{Acknowledgements}

The work was carried out under the HPC-EUROPA2 project (project number: 228398) with the support of the European Commission - Capacities Area - Research Infrastructures. This work made use of the facilities of HECToR, the UK's national highperformance computing service, which is provided by UoE HPCx Ltd at the University of Edinburgh, Cray Inc and NAG Ltd, and funded by the Office of Science and Technology through EPSRC's High End Computing Programme. Further access to the facilities of HECToR was provided through the HECToR Resource Allocation Panel, project number e174. The project was also funded under the HECToR Distributed Computational Science and Engineering (CSE) Service operated by NAG Ltd.

Finally, the work was also supported by the Ulysses-Ireland/France Research Visits Scheme, a programme for research visits between Ireland and France, jointly funded and administered by The Irish Research Council, the Irish Research Council for Science 
Engineering and Technology and Egide, the French agency for international mobility, with participation from the French Embassy in Ireland and Teagasc.

\section{Appendix A. Orr-Sommerfeld and Orr-Sommerfeld Squire equations}

We describe here the equations and the numerical method for the 3D modal analysis that is used directly in Section 2 and that forms the basis for the transient growth calculations in Section 3. In the base state, the interface is flat $(\eta=0)$, the flow is steady and unidirectional, $v=w=0, u=U_{0}(z)$, and the pressure is linear, $p=(d P / d L) x$, under a negative dimensionless pressure gradient, $d P / d L$. The solution for the laminar velocity profile is then

$$
U_{0}(z)= \begin{cases}U_{B}(z)=-\frac{R e}{2 m} z^{2}+A z, & 0 \leqslant z \leqslant h_{0}, \\ U_{T}(z)=-\frac{R e}{2}(z-1)^{2}+B(z-1), & h_{0} \leqslant z \leqslant 1\end{cases}
$$

The constants $A$ and $B$ are determined from continuity of velocity and shear stress at the interface:

$$
U_{B}\left(h_{0}\right)=U_{T}\left(h_{0}\right), \quad m U_{B}^{\prime}\left(h_{0}\right)=U_{T}^{\prime}\left(h_{0}\right) .
$$

As mentioned in Sections 2-3, we study the stability of the system by subjecting the base state to a small-amplitude three-dimensional perturbation. Each flow variable is expressed as a sum of the base state and the perturbation:

$$
\begin{array}{r}
\eta=h_{0}+\epsilon \eta_{0} \mathrm{e}^{\mathrm{i}(\alpha x+\beta y-\Omega t)}, \quad w=\epsilon \widetilde{w}(z) \mathrm{e}^{\mathrm{i}(\alpha x+\beta y-\Omega t)}, \quad \omega_{z}=\epsilon \widetilde{\omega}_{z}(z) \mathrm{e}^{\mathrm{i}(\alpha x+\beta y-\Omega t)}, \\
p=\frac{d P}{d L} x+\epsilon \widetilde{p}(z) \mathrm{e}^{\mathrm{i}(\alpha x+\beta y-\Omega t)} .
\end{array}
$$

Here $\epsilon$ is the infinitesimally small amplitude of the wave and $\eta_{0}$ is its phase (with $\left|\eta_{0}\right|=1$ ). Substituting Equations (A 3) into the equations of motion and boundary conditions, and dropping terms that are nonlinear in the perturbed variables, we get the following system of governing equations:

$$
\begin{aligned}
\mathrm{i} \alpha r \operatorname{Re}\left[\left(\widetilde{w}_{B}^{\prime \prime}-k^{2} \widetilde{w}_{B}\right)\left(U_{B}-c\right)-\widetilde{w}_{B} U_{B}^{\prime \prime}\right] & =m\left(\widetilde{w}_{B}^{\prime \prime \prime \prime}-2 k^{2} \widetilde{w}_{B}^{\prime \prime}+k^{4} \widetilde{w}_{B}\right), \\
\operatorname{ir} \operatorname{Re}\left[\alpha \widetilde{\omega}_{z B}\left(U_{B}-c\right)+\beta U_{B}^{\prime} \widetilde{w}_{B}\right] & =m\left(\widetilde{\omega}_{z B}^{\prime \prime}-k^{2} \widetilde{\omega}_{z}\right),
\end{aligned}
$$

in the bottom phase, with $k^{2}=\alpha^{2}+\beta^{2}$, and

$$
\begin{aligned}
\mathrm{i} \alpha R e\left[\left(\widetilde{w}_{T}^{\prime \prime}-k^{2} \widetilde{w}_{T}\right)\left(U_{T}-c\right)-\widetilde{w}_{T} U_{T}^{\prime \prime}\right] & =\widetilde{w}_{T}^{\prime \prime \prime \prime}-2 k^{2} \widetilde{w}_{T}^{\prime \prime}+k^{4} \widetilde{w}_{T}, \\
\mathrm{i} R e\left[\alpha \widetilde{\omega}_{z T}\left(U_{T}-c\right)+\beta U_{T}^{\prime} \widetilde{w}_{T}\right] & =\widetilde{\omega}_{z T}^{\prime \prime}-k^{2} \widetilde{\omega}_{z T},
\end{aligned}
$$

in the top phase. These are supplemented with the following no-slip and no-penetration boundary conditions:

$$
\widetilde{w}=\widetilde{w}^{\prime}=\widetilde{\omega}_{z}=0
$$

at the walls $z=0$ and $z=1$. In addition, matching conditions are prescribed at the interface $z=h_{0}$. In the streamwise direction, continuity of velocity and tangential stress and the jump condition in the normal stress imply the following relations:

$$
\begin{aligned}
\widetilde{w}_{B} & =\widetilde{w}_{T}, \\
\widetilde{w}_{B}^{\prime}+\eta_{0} U_{B}^{\prime} & =\widetilde{w}_{T}^{\prime}+\eta_{0} U_{T}^{\prime}, \quad \eta_{0}=\widetilde{w}_{B} /\left(c-U_{B}\right)=\widetilde{w}_{T} /\left(c-U_{T}\right), \\
m\left(\widetilde{w}_{B}^{\prime \prime}+k^{2} \widetilde{w}_{B}\right) & =\widetilde{w}_{T}^{\prime \prime}+k^{2} \widetilde{w}_{T},
\end{aligned}
$$




$$
\begin{aligned}
& \mathrm{i} \alpha r \operatorname{Re}\left[\widetilde{w}_{B}\left(c-U_{B}\right)+\widetilde{w}_{B} U_{B}^{\prime}\right]+m\left(\widetilde{w}_{B}^{\prime \prime \prime}-3 k^{2} \widetilde{w}_{B}\right) \\
& \quad=\mathrm{i} \alpha \operatorname{Re}\left[\widetilde{w}_{T}^{\prime}\left(c-U_{T}\right)+\widetilde{w}_{T} U_{T}^{\prime}\right]+\left(\widetilde{w}_{T}^{\prime \prime \prime}-3 k^{2} \widetilde{w}_{T}\right)+\mathcal{S} k^{4}\left[\frac{\widetilde{w}_{T}^{\prime}-\widetilde{w}_{B}^{\prime}}{\mathrm{i} \alpha\left(U_{B}^{\prime}-U_{T}^{\prime}\right)}\right]=0 .
\end{aligned}
$$

Finally, the same physical matching conditions applied to the spanwise direction give rise to the following relations:

$$
\begin{aligned}
\widetilde{\omega}_{z B}+\mathrm{i} \beta U_{B}^{\prime} \eta_{0} & =\widetilde{\omega}_{z T}+\mathrm{i} \beta U_{T}^{\prime} \eta_{0}, \\
m \widetilde{\omega}_{z B}^{\prime} & =\widetilde{\omega}_{z T} .
\end{aligned}
$$

Equations (A 4)-(A 6) constitute an eigenvalue problem for the velocities $\left(\widetilde{w}_{B}, \widetilde{w}_{T}\right)$ and vorticity components $\left(\widetilde{\omega}_{z B}, \widetilde{\omega}_{z T}\right)$, with eigenvalue $\lambda=-\mathrm{i} \alpha c=-\mathrm{i} \omega$.

We solve Equations (A 4)-(A 6) using the Chebyshev collocation method described by Boomkamp et al. (1997), wherein a trial solution involving the Chebyshev polynomials $T_{j}(\cdot)$ is proposed in each domain:

$$
\begin{array}{ll}
\widetilde{w}_{B}(z) \approx \sum_{j=0}^{N_{B}} a_{j} T_{j}\left(\eta_{B 1}\right), & \widetilde{\omega}_{z B}(z) \approx \sum_{j=0}^{N_{B}} b_{j} T_{j}\left(\eta_{B 2}\right), \\
\widetilde{w}_{T}(z) \approx \sum_{j=0}^{N_{T}} c_{j} T_{j}\left(\eta_{T 1}\right), & \widetilde{\omega}_{z T}(z) \approx \sum_{j=0}^{N_{T}} d_{j} T_{j}\left(\eta_{T 2}\right) ;
\end{array}
$$

this reduces the differential equations (A 4) to a finite-dimensional eigenvalue problem. The variables $\left(\eta_{B 1}, \eta_{B 2}, \eta_{T 1}, \eta_{T 2}\right)$ are linear transformations of the $z$-coordinate, whose range is confined to $[-1,1]$. The trial solution for $\left(\widetilde{w}_{B}, \widetilde{w}_{T}\right)$ is substituted into the differential equation (A 4$)$ and evaluated at $\left(N_{B}-3, N_{T}-3\right)$ interior points; similarly, the trial solution for $\left(\widetilde{\omega}_{z B}, \widetilde{\omega}_{z T}\right)$ is substituted into the differential equation (A 4) and evaluated at $\left(N_{B}-1, N_{T}-1\right)$ interior points. This gives $2\left(N_{B}+N_{T}\right)-8$ equations in $2\left(N_{B}+N_{T}\right)+4$ unknowns; the system is closed by evaluating the trial functions at the boundaries $z=0$ and $z=1$, and at the interface $z=h_{0}$ (12 further equations). In this way, a finite-dimensional analogue of Equations (A 4)-(A 6) is obtained:

$$
\boldsymbol{A v}=\lambda \boldsymbol{B} \boldsymbol{v}
$$

where $\boldsymbol{A}$ and $\boldsymbol{B}$ are $2\left(N_{B}+N_{T}+2\right) \times 2\left(N_{B}+N_{T}+2\right)$ complex matrices, and

$$
\boldsymbol{v}=\left(a_{0}, \cdots, a_{N_{B}}, b_{0}, \cdots, b_{N_{B}}, c_{0}, \cdots, c_{N_{T}}, d_{0}, \cdots, d_{N_{T}}\right)^{T}
$$

is a complex column-valued column vector. The eigenvalue $\lambda$ is obtained using a standard eigenvalue solver; the correctness of the implementation of the numerical scheme has been validated by computing the dispersion relation $\lambda(\alpha, \beta)$ for a given set of flow parameters, and comparing the result with the same dispersion relation computed by an different, independent method (Sahu \& Matar 2011); the results are identical.

\section{REFERENCES}

Azzopardi, B.J. 2003 Drops ina nnular two-phase flow. Intl J. Multiph. Flow 23 (suppl), $1-53$.

Barthelet, P., Charru, F. \& Fabre, J. 1995 Experimental study of interfacial long waves in a two-layer shear flow. J. Fluid Mech. 303, 23.

Batchelor, G. K. 1967 An Introduction to Fluid Dynamics. Cambridge University Press.

Boomkamp, P. A. M., Boersma, B. J., Miesen, R. H. M. \& v. Beijnon, G. 1997 A Chebyshev collocation method for solving two-phase flow stability problems. J. Comp. Phys 132, 191. 
Boomkamp, P. A. M. \& Miesen, R. H. M. 1996 Classification of instabilities in parallel two-phase flow. Int. J. Multiphase Flow 22, 67.

Boyd, J. P. 2001 Chebyshev and Fourier Spectral Methods. Dover.

Charru, FrançOIs \& Hinch, E 2000 Phase diagram of interfacial instabilities in a two-layer couette flow and mechanism of the long-wave instability. Journal of Fluid Mechanics 414, $195-223$.

Chomaz, Jean-Marc 2003 Fully nonlinear dynamics of parallel wakes. J. Fluid Mech. 495, $57-75$.

Couniron, A. \& Chomaz, J. M. 1999 Primary and secondary nonlinear global instability. Physica D 132, 428-456.

Craik, Alex DD 1969 The stability of plane couette flow with viscosity stratification. Journal of Fluid Mechanics 36 (04), 685-693.

Craik, A. D. D. 1985 Wave interactions and fluid flows. Cambridge, UK: Cambridge University Press.

Cristini, V., Guido, S., Alfani, A., Blawzdziewicz, J. \& Loewenberg, M. 2003 Drop breakup and fragment size distribution in shear flow. J. Rheol. 47, 1283-1298.

Delbende, I. \& Chomaz, J.-M. 1998 Nonlinear convective/absolute instabilities in parallel two-dimensional wakes. Phys. Fluids 10, 2724-2736.

Delbende, I., Chomaz, J.-M. \& Huerre, P. 1998 Absolute/convective instabilities in the Batchelor vortex: a numerical study of the linear impulse response. J. Fluid Mech. 355, 229-254.

Ding, H., Gilani, M. N. H. \& Spelt, P. D. M. 2010 Sliding, pinch-off and detachment of a droplet on a wall in shear flow. J. Fluid Mech. 644, 217-244.

Ding, H., Spelt, P. D M. \& Shu, C. 2007 Diffuse interface model for incompressible two-phase flows with large density ratios. J. Comput. Phys. 226, 2078.

Fuster, D., Agbaglah, G., Josserand, C., Popinet, S. \& Zaleski, S. 2009 Numerical simulation of droplets, bubbles, and waves: state of the art. Fluid Dyn. Res. 41, 065001.

Govindarajan, R. \& Sahu, K. C. 2014 Instabilities in viscosity-stratified flow. Annual Review of Fluid Mechanics 46, 331-353.

Gropp, W., Lusk, E. \& SkJellum, A. 1994 Using MPI: portable parallel programming with the message-passing interface. Cambridge, MA: MIT Press.

Huerre, P. 2000 Open shear flow instabilities. In Perspectives in Fluid Mechanics (ed. G. K. Batchelor, H. K. Moffatt \& M. G. Worster). Cambridge.

Huerre, P. \& Monkewitz, P. A. 1990 Local and global instability in spatially developing flows. Ann. Rev. Fluid Mech. 22, 473-537.

KING, M.R. \& MCCREADY, M.J. 2000 Weakly nonlinear simulation of planar stratified flows. Phys. Fluids 12, 92-102.

Lecoeur, N., Hale, C. P., Spelt, P. D. M. \& Hewitt, G. F. 2010 Visualization of droplet entrainment in turbulent stratified pipe flow. In 7th International Conference on Multiphase Flow ICMF 2010.

Marmottant, P. \& Villermaux, E. 2004 On spray formation. J. Fluid Mech. 498, 72-111.

Meland, R., Gran, I.R., Olsen, R. \& Munkejord, S. T. 2007 Reduction of parasitic currents in level-set calculations with a consistent discretization of the surface-tension force for the csf model. In Proceedings, 16th Australasian Fluid Mechanics Conference.

Miles, J. W. 1957 On the generation of surface waves by shear flows. J. Fluid Mech. 3, 185.

van Noorden, T. L., Boomkamp, P. A. M., Knapp, M. C. \& Verheggen, T. M. M. 1998 Transient growth in parallel two-phase flow: Analogies and differences with single-phase flow. Phys. Fluids 10, 2099.

Pavliotis, G. \& Stuart, A. M. 2008 Multiscale Methods. New York: Springer.

Russo, G. \& Smereka, P. 2000 A remark on computing distance functions. Computational Physics 163, 51.

Sahu, K. \& Matar, O. K. 2011 Three-dimensional convective and absolute instabilities in pressure-driven two-layer channel flow. Int. J. Multiphase Flow 37, 987.

Scardovelli, R. \& Zaleski, S. 1999 Direct numerical simulation of free-surface and interfacial flow. Annu. Rev. Fluid Mech. 31, 567.

Schmid, P. J. \& Henningson, D. S. 2001 Stability and Transition in Shear Flows. New York: Springer. 
Scott, D. M., NÁraigh, L. Ó, Bethune, I., Valluri, P. \& Spelt, P. D. M. $2013 a$ Performance enhancement and optimization of the TPLS and DIM two-phase flow solvers. Tech. Rep.. Edinburgh Parallel Computing Centre.

Scott, D. M., Náraigh, L. Ó, Bethune, I., Valluri, P. \& Spelt, P. D. M. $2013 b$ TPLS: High resolution direct numerical simulation (DNS) of two-phase flows. http://sourceforge.net/projects/tpls/.

Selvam, B, Talon, Laurent, Lesshafft, L \& Meiburg, E 2009 Convective/absolute instability in miscible core-annular flow. part 2. numerical simulations and nonlinear global modes. J. Fluid Mech. 618, 323-348.

Sussman, M. \& FAtemi, E. 1998 An efficient, interface-preserving level set redistancing algorithm and its application to interfacial incompressible flow. SIAM Journal on Scientific Computing 24, 1165-1191.

TAYLOR, G. I. 1953 Dispersion of soluble matter in solvent flowing slowly through a tube. Proc. R. Soc. Lond. A 219, 186-203.

Ó NÁraigh, L., Spelt, P. D. M., Matar, O. K. \& Zaki, T. A. 2011 Interfacial instability of turbulent two-phase stratified flow: Pressure-driven flow and thin liquid films. Int. J. Multiph. Flow 37, $812-830$.

Ó Náraigh, L., Spelt, P. D. M. \& Shaw, S. J. 2013 Absolute linear instability in laminar and turbulent gas/liquid two-layer channel flow. J. Fluid Mech. 714, 24.

Trefethen, N. L., Trefethen, A. E., Teddy, S. C. \& Driscoll, T. A. 1993 Hydrodynamic stability without eigenvalues. Science 561, 578.

Valluri, P., Spelt, P.D.M., Lawrence, C.J. \& Hewitt, G.F. 2007 Numerical simulation of the onset of slug initiation in laminar horizontal channel flow. Int. J. Multiphase Flow 34, 206.

Valluri, P., Ó Náraigh, L., Ding, H. \& Spelt, P. D. M. 2010 Linear and nonlinear spatiotemporal instability in laminar two-layer flows. J. Fluid Mech. 656, 458-480.

Yecko, P. \& ZAleski, S. 2005 Transient growth in two-phase mixing layers. J. Fluid Mech. 528, 43.

YeCKo, P., Zaleski, S. \& Fullana, J.-M. 2002 Viscous modes in two-phase mixing layers. Phys. Fluids 14, 4115.

Yiantsios, S. G. \& Higgins, B. G. 1988 Linear stability of plane Poiseuile flow of two superposed fluids. Phys. Fluids 31, 3225.

Yıн, C. S. 1967 Instability due to viscosity stratification. J. Fluid Mech. 27, 337. 